\title{
LYAPUNOV MAPS, SIMPLICIAL COMPLEXES AND THE STONE FUNCTOR
}

\author{
Joel W. Robbin and Dietmar A. Salamon \\ Department of Mathematics \\ University of Wisconsin-Madison \\ Madison, Wisconsin 53706, U.S.A. \\ and \\ Mathematics Institute \\ University of Warwick \\ Coventry, CV4 7AL, Great Britain
}

\section{INTRODUCTION}

Perhaps one of the most influential ideas in Smale's seminal 1967 paper [20] is that a general dynamical system should have a structure something like that of a gradient dynamical system. In pursuit of this idea Smale defined Axiom A No-Cycle systems and showed that they had three gradient like properties:

(1) Spectral Decomposition: The non-wandering set of such a system can be uniquely decomposed as a disjoint finite union

$$
\Omega=\Omega_{1} \cup \Omega_{2} \cup \cdots \Omega_{m}
$$

of closed transitive invariant subsets. In the case of a gradient dynamical system with non-degenerate critical points each of these subsets consists of a single critical point.

(2) Partial Ordering: The transitive closure of the relation

$$
W^{s}\left(\Omega_{i}\right) \cap W^{u}\left(\Omega_{j}\right) \neq \emptyset
$$

is a partial order. We denote this order by $\Omega_{i} \prec \Omega_{j}$. In the case of a MorseSmale gradient dynamical system (or more generally a system satisfying Smale's Axioms A and B) the ' $\Lambda$-lemma' of Palis [14] implies that it is not necessary to take the transitive closure:

$$
\Omega_{i} \prec \Omega_{j} \Longleftrightarrow W^{s}\left(\Omega_{i}\right) \cap W^{u}\left(\Omega_{j}\right) \neq \emptyset .
$$

(3) Filtration: Assume that the indexing of the $\Omega_{i}$ has been chosen so as to be consistent with the Smale partial order, that is

$$
\Omega_{i} \prec \Omega_{j} \Longrightarrow i \leq j .
$$

Then there is a filtration

$$
\emptyset=M_{0} \subset M_{1} \subset M_{2} \subset \cdots \subset M_{m}=M
$$


of the underlying manifold $M$ by positively invariant submanifolds with boundary with the property that

$$
\Omega_{i} \subset \operatorname{int}\left(M_{i} \backslash M_{i-1}\right) .
$$

In the case of a gradient dynamical system where the critical values $c_{i}$ corresponding to the critical points $\Omega_{i}$ are distinct and increase with $i$ the filtration may be constructed by taking sublevel sets

$$
M_{i}=h^{-1}\left(\left(-\infty, b_{i}\right]\right)
$$

where $b_{i}$ is a regular value of $h$ between $c_{i}$ and $c_{i+1}$ and $h$ is the height function whose gradient generates the dynamical system.

Conley [4] pointed out that the the foregoing theory can easily be generalized by focusing on the attractors of the dynamical system rather than the sets $\Omega_{i}$ of the spectral decomposition. Stone's theorem [23] (see also [12], [11]) is helpful in describing this generalization. According to this theorem any finite lattice is isomorphic to the lattice of lower sets of a finite partially ordered set (poset) $P$. In case of an Axiom A no-cycle dynamical system we may take

$$
P=\left\{\Omega_{1}, \Omega_{2}, \ldots, \Omega_{m}\right\}
$$

with the Smale order described above. Then the attractors of the dynamical system are precisely the sets

$$
A_{\alpha}=\bigcup_{p \in \alpha} W^{u}\left(\Omega_{p}\right)
$$

where $\alpha$ ranges over the lower sets of the poset $P$. The representation $\alpha \mapsto A_{\alpha}$ is an isomorophism of lattices:

$$
A_{\alpha \cap \beta}=A_{\alpha} \cap A_{\beta}, \quad A_{\alpha \cup \beta}=A_{\alpha} \cup A_{\beta}, \quad A_{\emptyset}=\emptyset, \quad A_{P}=M .
$$

In the case of a general dynamical system (on a compact metric space) the lattice of attractors need not be finite but for any finite sublattice analogs of Smale's three points continue to hold. In particular Franzosa [8] showed that such a finite sublattice admitted a system of neighborhoods $A_{\alpha} \subset N_{\alpha}$ satisfying

$$
N_{\alpha \cap \beta}=N_{\alpha} \cap N_{\beta}, \quad N_{\alpha \cup \beta}=N_{\alpha} \cup N_{\beta}, \quad N_{\emptyset}=\emptyset, \quad N_{P}=M .
$$

This generalizes Smale's filtrations. The neighborhoods $N_{\alpha}$ are used to define topological invariants of the dynamical system as in [3] (see also [13], [15], [17]). These invariants have proved quite useful in solving problems which at first glance appear to be far removed from the theory. (See for example [5], [18], [22].)

Conley [4] pointed out the connection between attractors and Lyapunov functions. Suppose that $f^{t}$ is a dynamical system on a space $M$. A Lyapunov function for a dynamical system is a continuous function $\theta: M \rightarrow[0,1]$ such that the sets $A=\theta^{-1}(0)$ and $A^{*}=\theta^{-1}(1)$ are invariant and $\theta$ is strictly decreasing along orbits not in these two sets. It is not difficult to prove (see $\S 1$ ) that $A$ is an attractor, $A^{*}$ is its dual repeller, and that every attractor repeller pair may be defined by a Lyapunov function in this way. Note that the Lyapunov functions form a lattice 
(the lattice operations are the pointwise max and min of functions) and that the map $\theta \mapsto \theta^{-1}(0)$ is an anti-homomorphism of lattices.

In this paper we show how to construct a lattice of Lyapunov functions

$$
\theta_{\alpha \cap \beta}=\theta_{\alpha} \vee \theta_{\beta}, \quad \theta_{\alpha \cup \beta}=\theta_{\alpha} \wedge \theta_{\beta}, \quad \theta_{\emptyset}=1, \quad \theta_{P}=0
$$

which defines a given lattice of attractors in the sense that

$$
A_{\alpha}=\theta_{\alpha}^{-1}(0)
$$

Franzosa's theorem mentioned above is an easy corollary for we may define the neighborhoods $N_{\alpha}$ by

$$
N_{\alpha}=\theta_{\alpha}^{-1}([0,1 / 2])
$$

It is of interest to describe the system $\left\{\theta_{\alpha}\right\}_{\alpha}$ of Lyapunov functions in terms of a map $\psi: M \rightarrow K(P)$ to a certain simplicial complex $K(P)$ which depends on the partially ordered set $P$. We term this map $\psi$ a Lyapunov map. This complex $K(P)$ is well-known to topologists; the earliest explicit references in the literature which we were able to find are [7] and [19]. Folkman [7] used it to solve a problem of Rota [16]. The observation which relates $\psi$ and $\left\{\theta_{\alpha}\right\}_{\alpha}$ is that $K(P)$ may be described as the space of lattice homomorphisms from the lattice $L$ of lower sets of $P$ to the interval $[0,1]$. In this way the complex $K(P)$ appears as a kind of extension of the Stone space of $L$. (The Stone space is by definition the space of all lattice homomorphisms from $L$ to the two point lattice $\{0,1\}$ : Stone's theorem is that it is isomorphic to $P$.)

Our Lyapunov map evidently has an intimate connection with the as yet unpublished theorem of Cohen, Jones, and Segal [2]. A Morse-Smale gradient flow determines a topological category $\mathcal{C}$ as follows: the set of objects of $\mathcal{C}$ is the set $P$ of critical points of the flow and the set $\operatorname{Mor}(q, p)$ of morphisms from $q$ to $p$ is the set of all sequences $\left(\gamma_{1}, \gamma_{2}, \ldots, \gamma_{\ell}\right)$ of orbits where $\gamma_{k}$ runs from the critical point $p_{k-1}$ to the critical point $p_{k}$ and $p_{0}=q$ and $p_{\ell}=p$. The composition of morphisms is given by concatenating the sequences. Note that $\operatorname{Mor}(q, p) \neq \emptyset$ if and only if $p \prec q$ in the Smale order. There is a natural 'forgetful functor' $\pi: \mathcal{C} \rightarrow P$ which is the identity on the set of objects. Here the poset $P$ is identified with the category whose objects are the elements of $P$ and with one morphism $q \rightarrow p$ for every relation $p \prec q$.

In [19], Segal defines the classifying space $B \mathcal{C}$ of a topological category $\mathcal{C}$. This construction has the classifying space $B G$ of a group as a special case. The simplicial complex $K(P)$ appears as the classifying space of the category associated to the poset $P$. The theorem of Cohen, Jones, and Segal asserts that the classifying space $B \mathcal{C}$ of the category $\mathcal{C}$ of a Morse-Smale gradient flow is homeomorphic to the underlying manifold $M$. Presumably the homeomorphism $\phi: M \rightarrow B \mathcal{C}$ can be chosen so that the composition $\psi=B \pi \circ \phi$ is a Lyapunov map. Here $B \pi: B \mathcal{C} \rightarrow$ $K(P)$ is the map of classifying spaces induced by the functor $\pi: \mathcal{C} \rightarrow P$.

In $\S 6-8$ we treat the connection matrices of Franzosa [10] with our methods.

Thanks to Ed Fadell, Mo Hirsch, Sufian Husseini, John Jones, Colin Rourke, and Bruce Westbury for helpful discussions. 


\section{$\S 0$ Notation}

Throughout $(M, d)$ denotes a compact metric space and $f$ denotes a dynamical system on $M$. We consider simultaneously the case of discrete time $\mathbb{T}=\mathbb{Z}$ and continuous time $\mathbb{T}=\mathbb{R}$. In either case the dynamical system $f$ is a group homomorphism

$$
\mathbb{T} \rightarrow \operatorname{Homeo}(M): t \mapsto f^{t}
$$

from the time line $\mathbb{T}$ to the group of self homeomorphisms of $M$. In the case of continuous time we impose the additional condition that the evaluation map

$$
\mathbb{R} \times M \rightarrow M:(t, x) \mapsto f^{t}(x)
$$

is continuous. Note that in the discrete case the letter $f$ is used ambiguously to denote both the group homomorphism $t \mapsto f^{t}$ and its generator $f^{1}$. We extend this ambiguity to the continuous time case so that $f^{-1}$ denotes the flow $t \mapsto f^{-t}$.

For any invariant subset $A \subset M$ we define the stable and unstable manifold of $A$ in $M$ by

$$
\begin{aligned}
& W^{s}(A)=\left\{x \in M \mid \lim _{t \rightarrow \infty} d\left(f^{t}(x), A\right)=0\right\}, \\
& W^{u}(A)=\left\{x \in M \mid \lim _{t \rightarrow-\infty} d\left(f^{t}(x), A\right)=0\right\} .
\end{aligned}
$$

All lattices have a minimal and a maximal element (both are necessarily unique), lattice homomorphisms are required to preserve these elements, and sublattices are required to contain them. The word poset abbreviates partially ordered set. For lattices $L, L^{\prime}$ and posets $P, P^{\prime}$ define

$$
\begin{aligned}
\operatorname{Hom}\left(L, L^{\prime}\right) & =\left\{\text { lattice homomorphisms } L \rightarrow L^{\prime}\right\} \\
\operatorname{Hom}^{*}(L, M) & =\left\{\text { lattice anti-homomorphisms } L \rightarrow L^{\prime}\right\} \\
\operatorname{Ord}\left(P, P^{\prime}\right) & =\left\{\text { order preserving maps } P \rightarrow P^{\prime}\right\} \\
\operatorname{Ord}^{*}\left(P, P^{\prime}\right) & =\left\{\text { order reversing maps } P \rightarrow P^{\prime}\right\} .
\end{aligned}
$$

Denote by $I$ the closed unit interval and by $\partial I$ its boundary:

$$
I=[0,1], \quad \partial I=\{0,1\} .
$$

Both of these are lattices. The lattice operations are min (meet) and max (join). We write

$$
a \wedge b=\min (a, b), \quad a \vee b=\max (a, b)
$$

The equation

$$
a+b=a \wedge b+a \vee b
$$

is helpful.

What others call a Morse decomposition we call an attractor network. The reason is that in the generality considered here, the Morse decomposition (unlike the spectral decomposition of Smale [20]) is not a decomposition of anything. What Franzosa calls attracting interval we call lower set. This agrees more with standard usage in lattice theory (see [11]). Finally, we often use anti-homomorphisms where others use homomorphisms, for example, in the definition of the Stone space. The reason is that these often relate to Lyapunov functions and we require Lyapunov functions to decrease along orbits. 


\section{$\S 1$ The LATtice of ATTRACTORS}

An attractor for $f$ in $M$ is a compact invariant set $A$ which admits a neighborhood $U$, called an attracting neighborhood of $A$, such that

$$
f^{t}(\operatorname{cl}(U)) \subset \operatorname{int}(U) \quad \text { for } \quad t>0
$$

and

$$
A=\bigcap_{t \geq 0} f^{t}(U)
$$

Our definition of attractor agrees with the definition in [3]: It is easy to show (using Proposition 1.4 below) that an attractor for the discrete time system with generator $f^{t_{0}}$ with $t_{0}>0$ is an attractor for $f$. We denote by $\mathcal{A}(M, f)$ the set of attractors for $f$ in $M$. A repeller for $f$ is an attractor for the time reversed dynamical system $f^{-1}$ so that $\mathcal{A}\left(M, f^{-1}\right)$ denotes the set of repellers for $f$ in $M$.

Proposition 1.1. (i) The set $\mathcal{A}(M, f)$ forms a finite or countably infinite lattice.

(ii) For every attractor $A \in \mathcal{A}(M, f)$ the set

$$
A^{*}=M \backslash W^{s}(A)
$$

is a repeller, called the complementary repeller of $A$.

(iii) The map

$$
\mathcal{A}(M, f) \rightarrow \mathcal{A}\left(M, f^{-1}\right): A \mapsto A^{*}
$$

is a lattice anti-isomomorphism. Its inverse is given by the formula

$$
A^{*} \mapsto A=M \backslash W^{u}\left(A^{*}\right)
$$

An attractor network for $(M, f)$ is a finite sublattice $\mathcal{A}$ of $\mathcal{A}(M, f)$. By Stone's representation theorem [12] the lattice $\mathcal{A}$ is isomorphic to the lattice of lower sets of some finite poset $(P, \preccurlyeq)$. We examine what this means.

A subset $\alpha \subset P$ is called a lower set if $q \in \alpha$ whenever $p \in \alpha$ and $q \preccurlyeq p$. The collection of lower sets in $P$ forms a lattice denoted by $\mathcal{L}(P)$. Note that for any two distinct points in $P$ there exists a lower set which contains one and not the other. Now Stone's theorem says that every finite distributive lattice $L$ is isomorphic to a lattice of the form $\mathcal{L}(P)$. The poset $P$ is uniquely determined (up to order isomorphism) by the lattice $L$. As a notational device we index the attractors of our attractor network by the lower sets of some finite poset $P$. Hence we call a lattice homomorphism

$$
\mathcal{L}(P) \rightarrow \mathcal{A}(M, f): \alpha \mapsto A_{\alpha}
$$

where $P$ be a finite poset an attractor network indexed by $P$. The network satisfies

$$
A_{\alpha \cap \beta}=A_{\alpha} \cap A_{\beta}, \quad A_{\alpha \cup \beta}=A_{\alpha} \cup A_{\beta}, \quad A_{\emptyset}=\emptyset, \quad A_{P}=M .
$$

We do not require the homomorphism $\alpha \rightarrow A_{\alpha}$ to be injective, but in all our arguments we may assume this without loss of generality. 
Theorem 1.2. Let $\left\{A_{\alpha}\right\}_{\alpha \in \mathcal{L}(P)}$ be an attractor network indexed by $P$. Then there exists a unique collection of compact isolated invariant sets $\Lambda_{p} \subset M$ indexed by $p \in P$ such that

$$
A_{\alpha}=\bigcup_{p \in \alpha} W^{u}\left(\Lambda_{p}\right), \quad A_{\alpha}^{*}=\bigcup_{q \notin \alpha} W^{s}\left(\Lambda_{q}\right), \quad W^{u}\left(\Lambda_{p}\right) \cap W^{s}\left(\Lambda_{p}\right)=\Lambda_{p} .
$$

The sets $\Lambda_{p}$ are called the basic sets of the attractor network.

In case the index set $P$ has been chosen so that $A_{\alpha} \neq A_{\beta}$ for $\alpha \neq \beta$, the basic sets $\Lambda_{p}$ are nonempty and both families $\left\{W^{u}\left(\Lambda_{p}\right)\right\}_{p \in P}$ and $\left\{W^{s}\left(\Lambda_{p}\right)\right\}_{p \in P}$ partition $M$ :

$$
\begin{gathered}
M=\bigcup_{p \in P} W^{u}\left(\Lambda_{p}\right)=\bigcup_{p \in P} W^{s}\left(\Lambda_{p}\right) \\
W^{u}\left(\Lambda_{p}\right) \cap W^{u}\left(\Lambda_{q}\right)=W^{s}\left(\Lambda_{p}\right) \cap W^{s}\left(\Lambda_{q}\right)=\emptyset
\end{gathered}
$$

for $p \neq q$. Moreover, in this case there is no connecting orbit from $\Lambda_{q}$ to $\Lambda_{p}$ unless $p \preccurlyeq q$. Therefore the basic sets $\Lambda_{p}$ satisfy the no cycle condition

$$
M \backslash \bigcup_{p \in P} \Lambda_{p}=\bigcup_{p \preccurlyeq q, p \neq q} W^{u}\left(\Lambda_{q}\right) \cap W^{s}\left(\Lambda_{p}\right) .
$$

Conversely, every such collection of isolated invariant sets determines an attractor network.

Theorem 1.3. Let $\left\{\Lambda_{p}\right\}_{p \in P}$ be a finite collection of disjoint nonempty isolated invariant sets indexed by a partially ordered set $P$ which satisfy the no cycle condition. Then $A_{\alpha}$ as defined in Theorem 1.2 is an attractor in $M$ for every lower set $\alpha \in \mathcal{L}(P)$.

The archetypal example is the time-one map $f$ of the gradient flow of a Morse function on a compact manifold $M$. In this situation the lattice $\mathcal{A}(M, f)$ of attractors is finite, the index poset $P$ is the set of critical points, and $\Lambda_{p}=\{p\}$. The order $\preccurlyeq$ on $P$ is the smallest partial order extending the relation $p \preccurlyeq 0 q$ iff there is a flow line from $q$ to $p$. If the flow is Morse-Smale (i.e. stable and unstable manifolds intersect transversally) then the relations $\preccurlyeq_{0}$ and $\preccurlyeq$ are the same and the attractors are precisely the unions of closures of unstable manifolds. However, in the case of the gradient flow on the 2-torus with two saddle connections $\preccurlyeq_{0}$ is not a partial order and the closure of the unstable manifold of the upper saddle is not an attractor.

Proof of Proposition 1.1. We prove statement (i). It is routine to show that if $U_{i}$ is an attracting neighborhood for $A_{i}, i=1,2$, then $U_{1} \cup U_{2}$ is an attracting neighborhood of $A_{1} \cup A_{2}$ and $U_{1} \cap U_{2}$ is an attracting neighborhood of $A_{1} \cap A_{2}$. If $U$ is an attracting neighborhood for $A$ then any open set $V$ with $f(\operatorname{cl}(U)) \subset V \subset U$ is also an attracting neighborhood for $A$. This neighborhood can be chosen to be a finite union of the elements of a countable base of the topology of $M$. Thus $\mathcal{A}(M, f)$ is at most countably infinite.

We prove statement (ii). Given $A \in \mathcal{A}(M, f)$ we first show that $W^{s}(A)$ is an open neighborhood of $A$. To see this let $U$ be an attracting neighborhood for $A$. Then 
the sets $f^{t}(\operatorname{cl}(U))$ form a decreasing collection of compact sets whose intersection is $A$. Hence for every $\varepsilon>0$

$$
A \subset f^{t}(\operatorname{cl}(U)) \subset\{x \in M \mid d(x, A)<\varepsilon\}
$$

provided that $t$ is sufficiently large. It follows that $U \subset W^{s}(A)$ and therefore $x \in W^{s}(A)$ if and only if $f^{t}(x) \in U$ for some $t \geq 0$. We conclude that $W^{s}(A)$ is open and $U^{*}=M \backslash U$ is an attracting neighborhood of $A^{*}=M \backslash W^{s}(A)$ with respect to $f^{-1}$.

We prove statement (iii). Since

$$
W^{s}\left(A_{1}\right) \cup W^{s}\left(A_{2}\right)=W^{s}\left(A_{1} \cup A_{2}\right), \quad W^{s}\left(A_{1}\right) \cap W^{s}\left(A_{2}\right)=W^{s}\left(A_{1} \cap A_{2}\right),
$$

the map $A \mapsto A^{*}$ is a lattice anti-homomorphism:

$$
\left(A_{1} \cup A_{2}\right)^{*}=A_{1}^{*} \cap A_{2}^{*}, \quad\left(A_{1} \cap A_{2}\right)^{*}=A_{1}^{*} \cup A_{2}^{*} .
$$

That $A=M \backslash W^{u}\left(A^{*}\right)$ follows from the fact that every orbit not in $A \cup A^{*}$ contains exactly one point in $U \backslash f(U)$ in the discrete time case and exactly one point in $\partial U$ in the continuous time case.

Proposition 1.4. Let $(A, R)$ be a pair of compact invariant sets in $M$. Then the following conditions are equivalent:

(1) The ambient space $M$ decomposes as the disjoint union

$$
M=A \cup\left(W^{s}(A) \cap W^{u}(R)\right) \cup R .
$$

(2) There exists a continuous function $\theta: M \rightarrow[0,1]$ such that

$$
A=\theta^{-1}(0), \quad R=\theta^{-1}(1),
$$

and

$$
\theta\left(f^{t}(x)\right)<\theta(x) \quad \text { for } \quad x \in M \backslash(A \cup R) \quad \text { and } \quad t>0 \text {. }
$$

(3) The set $A$ is an attractor and $R=A^{*}$ is its complementary repeller.

Such a pair $(A, R)$ is called an attractor-repeller pair and a function $\theta$ as in (2) is called a Lyapunov function for it.

Proof. The implication (3) implies (1) is obvious and for (2) implies (3) the required attracting neighborhood of $A$ is $\theta^{-1}([0,1 / 2])$. Hence we need only prove (1) implies (2). Our construction is a modification of the argument of Conley [4] which enables us to obtain smooth Lyapunov functions when $(M, f)$ is a smooth dynamical system.

Let $U$ be a neighborhood of $A$ such that $\operatorname{cl}(U) \cap R=\emptyset$. We claim that there exists an open neighborhood $V$ of $A$ such that

$$
f^{t}(x) \in U \quad \text { for all } t \geq 0, \quad x \in V .
$$

Otherwise there would exist sequences $x_{\nu}$ converging to $A$ and $t_{\nu} \geq 1$ such that $f^{t_{\nu}}\left(x_{\nu}\right) \notin U$ and $f^{s}\left(x_{\nu}\right) \in U$ for $0 \leq s<t_{\nu}$. Passing to a subsequence we may assume that $f^{t_{\nu}}\left(x_{\nu}\right)$ converges to $y \in f(\operatorname{cl}(U)) \backslash U$. So it follows that $t_{\nu}$ tends 
to infinity since otherwise $y \in A$. Hence $f^{-s}(y) \in \operatorname{cl}(U)$ for every $s \geq 0$. So $y \in M \backslash(A \cup R)$ but $y \notin W^{u}(R)$, a contradiction.

Now let $W$ be an open neighborhood of $R$ such that $\operatorname{cl}(W) \cap \operatorname{cl}(U)=\emptyset$. Then there exists a $T>0$ such that

$$
f^{t}(x) \in U \quad \text { for all } \quad t \geq T, \quad x \notin W .
$$

Otherwise there would exist a sequence $x_{\nu} \in M \backslash W$ and a sequence $T_{\nu}>0$ converging to $\infty$ such that $f^{T_{\nu}}\left(x_{\nu}\right) \notin U$. Hence $f^{t}\left(x_{\nu}\right) \notin V$ for $0 \leq t \leq T_{\nu}$. Assume without loss of generality that $x_{\nu}$ converges to $x \in M$. Then $x \notin W$ and $f^{t}(x) \notin V$ for every $t \geq 0$. Hence $x \notin R \cup W^{s}(A)$, a contradiction.

Let $\eta: M \rightarrow[0,1]$ be a continuous function with $\operatorname{cl}(W)=\eta^{-1}(1)$ and $\operatorname{cl}(U)=$ $\eta^{-1}(0)$. Then

$$
\eta(x)<1 \quad \Longrightarrow \quad \eta\left(f^{s}(x)\right)=0 \quad \text { for } s \geq T \text {. }
$$

At this point we consider the discrete time case $\mathbb{T}=\mathbb{Z}$. Define

$$
\rho(x)=\frac{1}{T} \sum_{k=0}^{T-1} \eta\left(f^{k}(x)\right)
$$

Then

$$
\rho(f(x))-\rho(x)=\frac{1}{T}\left(\eta\left(f^{T}(x)\right)-\eta(x)\right) \leq 0 .
$$

The desired Lyapunov function is

$$
\theta(x)=\sum_{k=-\infty}^{+\infty} c_{k} \rho\left(f^{k}(x)\right), \quad \sum_{k=-\infty}^{+\infty} c_{k}=1 .
$$

The numbers $c_{k}$ are chosen to be positive and to decay sufficiently rapidly so that the series converges uniformly. For $x \in M \backslash(A \cup R)$ there exists a $k$ with $\rho\left(f^{k+1}(x)\right)<$ $\rho\left(f^{k}(x)\right)$ since $\rho\left(f^{k}(x)\right)=1$ for $k \approx-\infty$ and $\rho\left(f^{k}(x)\right)=0$ for $k \approx \infty$. Hence at least one term in the sum defining $\theta(f(x))$ is strictly smaller than the corresponding term in $\theta(x)$. So $\theta(f(x))<\theta(x)$.

Now we consider the continuous time case $\mathbb{T}=\mathbb{R}$. Define

$$
\rho(x)=\int_{0}^{\infty} \beta(s) \eta\left(f^{s}(x)\right) d s .
$$

Here $\beta: \mathbb{R} \rightarrow \mathbb{R}$ is a smooth nonincreasing cutoff function of mean value 1 such that $\beta(t)=c>0$ for $t \leq T$ and $\beta(t)=0$ for $t \geq T+1$. The function $t \mapsto \rho\left(f^{t}(x)\right)$ is continuously differentiable and

$$
\dot{\rho}(x)=\left.\frac{d}{d t}\right|_{t=0} \rho\left(f^{t}(x)\right)=-c \eta(x)-\int_{T}^{T+1} \beta^{\prime}(s) \eta\left(f^{s}(x)\right) d s \leq 0 .
$$

The desired Lyapunov function is

$$
\theta(x)=\int_{-\infty}^{+\infty} c(s) \rho\left(f^{s}(x)\right) d s, \quad \int_{-\infty}^{+\infty} c(s) d s=1 .
$$


The function $c(s)$ is chosen to be positive and to decay sufficiently rapidly so that the integral converges uniformly. If $c(s)$ decays sufficiently rapidly then the function $t \mapsto \theta\left(f^{t}(x)\right)$ is continuously differentiable and

$$
\dot{\theta}(x)=\left.\frac{d}{d t}\right|_{t=0} \theta\left(f^{t}(x)\right)=\int_{-\infty}^{+\infty} c(s) \dot{\rho}\left(f^{s}(x)\right) d s
$$

is continuous. For $x \in M \backslash(A \cup R)$ there exists an $s \in \mathbb{R}$ with $\dot{\rho}\left(f^{s}(x)\right)<0$ since $\rho\left(f^{s}(x)\right)$ is not constant. Hence $\dot{\theta}<0$ on $M \backslash(A \cup R)$.

Remarks. i) The discrete time case in Proposition 1.4 can also be proved by applying the continuous time case to the suspension.

ii) If $(A, R)$ is an attractor-repeller pair for a smooth dynamical system $f$ on a smooth manifold $M$ then the function $\eta$ in the proof of Proposition 1.4 can be chosen smooth. It follows that the Lyapunov function $\theta$ is smooth for a suitable choice of the function $c(s)$. Note that the function $c(s)$ may be chosen constant on the intervals between integers.

Corollary 1.5. Let $A \in \mathcal{A}(M, f)$ be an attractor and let $M^{\prime} \subset M$ be a compact subset invariant under $f$. Then $A^{\prime}=A \cap M^{\prime}$ is an attractor for $f$ in $M^{\prime}$ with complementary repeller $R^{\prime}=A^{*} \cap M^{\prime}$.

Proof. The pair $\left(A^{\prime}, R^{\prime}\right)$ obviously satisfies condition (1) in Proposition 1.4 and is therefore an attractor-repeller pair in $M^{\prime}$.

Proof of Theorem 1.2. Given $p \in P$ there are lower sets $\alpha, \beta \in \mathcal{L}(P)$ with $\beta=$ $\alpha \cup\{p\}$ (for instance take $\beta=\{q \in P: q \preccurlyeq p\}$ ). The conclusion of the theorem requires that

$$
\Lambda_{p}=A_{\beta} \cap A_{\alpha}^{*}
$$

In order to take this as a definiton of $\Lambda_{p}$ we have to show that the right hand side is independent of the choice of $\beta$. We actually show more:

Lemma 1.6. For any $\alpha, \beta \in \mathcal{L}(P)$ the set

$$
\Lambda_{\beta \backslash \alpha}=A_{\beta} \cap A_{\alpha}^{*}
$$

is well defined.

Proof of Lemma 1.6. We must show that $A_{\beta_{1}} \cap A_{\alpha_{1}}^{*}=A_{\beta_{2}} \cap A_{\alpha_{2}}^{*}$ for $\alpha_{i}, \beta_{i} \in \mathcal{L}(P)$ with $\beta_{1} \backslash \alpha_{1}=\beta_{2} \backslash \alpha_{2}$. Without loss of generality assume $\beta_{1} \subset \beta_{2}$ and $\alpha_{1} \subset \alpha_{2}$. Then $\beta_{2}=\beta_{1} \cup \alpha_{2}$ and hence $A_{\beta_{2}}=A_{\beta_{1}} \cup A_{\alpha_{2}}$. Also $\alpha_{1}=\beta_{1} \cap \alpha_{2}$ and hence $A_{\alpha_{1}}^{*}=A_{\beta_{1}}^{*} \cup A_{\alpha_{2}}^{*}$. It follows that

$$
\begin{aligned}
A_{\beta_{2}} \cap A_{\alpha_{2}}^{*} & =\left(A_{\beta_{1}} \cup A_{\alpha_{2}}\right) \cap A_{\alpha_{2}}^{*} \\
& =A_{\beta_{1}} \cap A_{\alpha_{2}}^{*} \\
& =A_{\beta_{1}} \cap\left(A_{\beta_{1}}^{*} \cup A_{\alpha_{2}}^{*}\right) \\
& =A_{\beta_{1}} \cap A_{\alpha_{1}}^{*} .
\end{aligned}
$$

This proves that $\Lambda_{\beta \backslash \alpha}$, and in particular $\Lambda_{p}=\Lambda_{\{p\}}$ is well defined. 
The intersection $\Lambda$ of an attractor $A$ and a repeller $R$ is always an isolated invariant set. An isolating neighborhood for $\Lambda$ is $U \cap V$ where $U$ is an attracting neighborhood of $A$ and $V$ is a repelling (that is attracting for $f^{-1}$ ) neighborhood of $R$.

For any invariant set $\Lambda$ contained in an attractor $A$ the unstable manifold $W^{u}(\Lambda)$ is also contained in $A$. Hence

$$
\bigcup_{p \in \alpha} W^{u}\left(\Lambda_{p}\right) \subset A_{\alpha}
$$

for every lower set $\alpha \in \mathcal{L}(P)$. To prove the converse inclusion it suffices to show that

$$
A_{\beta} \backslash W^{u}\left(\Lambda_{p}\right)=A_{\alpha}
$$

whenever $\alpha, \beta \in \mathcal{L}(P)$ are lower sets with $\beta=\alpha \cup\{p\}$. This equation says that $\Lambda_{p}$ is the complementary repeller of $A_{\alpha}$ in $A_{\beta}$. Now $A_{\alpha}^{*}$ is the complementary repeller of $A_{\alpha}$ in $M$ and hence it follows from Corollary 1.5 that $\Lambda_{p}=A_{\beta} \cap A_{\alpha}^{*}$ is the complementary repeller of $A_{\alpha}$ in $A_{\beta}$ as required. Note that this also shows that $\Lambda_{p} \neq \emptyset$ since $A_{\alpha} \neq A_{\beta}$ and hence the complementary repeller of $A_{\alpha}$ in $A_{\beta}$ is nonempty. Replacing $f$ by $f^{-1}$ we see that

$$
A_{\alpha}^{*}=\bigcup_{q \notin \alpha} W^{s}\left(\Lambda_{q}\right) \text {. }
$$

Note that we have also shown that the isolated invariant set of Lemma 1.6 is given by

$$
\Lambda_{\beta \backslash \alpha}=\bigcup_{p \in \beta, q \notin \alpha} W^{u}\left(\Lambda_{p}\right) \cap W^{s}\left(\Lambda_{q}\right) .
$$

Proof of Theorem 1.3. The no-cycle condition implies that

$$
M=\bigcup_{p \in P} W^{u}\left(\Lambda_{p}\right)=\bigcup_{p \in P} W^{s}\left(\Lambda_{p}\right) .
$$

We prove by induction that the invariant sets

$$
A_{\alpha}=\bigcup_{p \in \alpha} W^{u}\left(\Lambda_{p}\right), \quad A_{\alpha}^{*}=\bigcup_{q \notin \alpha} W^{s}\left(\Lambda_{q}\right)
$$

are closed and hence compact for every lower set $\alpha \in \mathcal{L}(P)$.

First let $p \in P$ be a maximal element and suppose that $A_{P \backslash\{p\}}=M \backslash W^{u}\left(\Lambda_{p}\right)$ is not closed. Then there exists a sequence $x_{\nu} \notin W^{u}\left(\Lambda_{p}\right)$ converging to $x \in W^{u}\left(\Lambda_{p}\right)$. Take an open neighborhood $U$ of $\Lambda_{p}$ such that $\operatorname{cl}(U) \cap \Lambda_{q}=\emptyset$ for $q \neq p$. Then there exists a $t_{0} \geq 0$ such that $f^{-s}(x) \in U$ for all $s \geq t_{0}$. It follows that the sequence $t_{\nu} \in \mathbb{N}$ defined by

$$
f^{-s}\left(x_{\nu}\right) \in U \quad \text { for } \quad t_{0} \leq s<t_{\nu}, \quad f^{-t_{\nu}}\left(x_{\nu}\right) \notin U
$$

tends to infinity. Passing to a subsequence we may assume that $f^{-t_{\nu}}\left(x_{\nu}\right)$ converges to $y \notin U$. Since $f^{s}(y) \in \operatorname{cl}(U)$ for $s \geq 0$ it follows that $y \in W^{s}\left(\Lambda_{p}\right) \backslash \Lambda_{p}$. Thus there exists a $q \in P \backslash\{p\}$ with $p \preccurlyeq q$ and $p$ is not maximal.

Thus we have shown that $A_{P \backslash\{p\}}$ is closed for every maximal element $p \in P$. Replace $P$ by $P^{\prime}=P \backslash\{p\}$ and $M$ by $A_{P^{\prime}}$. Then it follows by induction that $A_{\alpha}$ is a compact invariant set for every lower set $\alpha \in \mathcal{L}(P)$. Replacing $f$ by $f^{-1}$ we obtain that $A_{\alpha}^{*}$ is a compact invariant set as well. By the no cycle condition the sets $A=A_{\alpha}$ and $R=A_{\alpha}^{*}$ satisfy condition (1) in Proposition 1.4. Hence $A_{\alpha}$ is an attractor in $M$ with complementary repeller $A_{\alpha}^{*}$. 


\section{$\S 2$ The Stone FunCtor}

Stone's theorem [23], [12], [11] asserts that every finite distributive lattice is isomorphic to the lattice of lower sets of some poset $P$. Moreover the isomorphism is "natural". In this section we explain and generalize Stone's theorem.

The Stone functor is a contravariant functor from the category of finite lattices to the category of finite posets. It assigns to each lattice $L$ the space $\operatorname{Hom}^{*}(L, \partial I)$ of lattice anti-homomorphisms from $L$ to the two-point lattice and to each lattice homomorphism $F \in \operatorname{Hom}(L, M)$ the map

$$
F^{*}: \operatorname{Hom}^{*}(M, \partial I) \rightarrow \operatorname{Hom}^{*}(L, \partial I), \quad F^{*} h=h \circ F
$$

induced by $F$. The space $\operatorname{Hom}^{*}(L, \partial I)$ is called the Stone space of the lattice $L$. It is partially ordered by $h \preccurlyeq k$ iff $h(\alpha) \leq k(\alpha)$ for all $\alpha \in L$.

The operation $\mathcal{L}$ defined in the previous section can also be viewed as a functor. It assigns to the poset $P$ the lattice $\mathcal{L}(P)$ of lower sets of $P$ and to the order preserving map $f: P \rightarrow Q$ the lattice homomorphism

$$
\mathcal{L}(f)=f^{-1}: \mathcal{L}(Q) \rightarrow \mathcal{L}(P)
$$

The lattice $\mathcal{L}(P)$ is a distributive lattice since its elements are sets and the lattice operations are intersection and union. There is a natural pairing

$$
\Theta: P \times \mathcal{L}(P) \rightarrow \partial I
$$

defined by

$$
\Theta(p, \alpha)= \begin{cases}0, & p \in \alpha, \\ 1, & p \notin \alpha .\end{cases}
$$

We introduce the notation

$$
\Theta(p, \alpha)=\Theta^{p}(\alpha)=\Theta_{\alpha}(p)
$$

The reader can check that $\Theta^{p}: \mathcal{L}(P) \rightarrow \partial I$ is a lattice anti-homomorphism and is therefore an element of the Stone space. The map $\Theta_{\alpha}: P \rightarrow \partial I$ is order preserving. The set $\operatorname{Ord}(P, \partial I)$ of order preserving maps is a lattice: the lattice operations are pointwise max and min. The pairing $\Theta$ identifies the lattice $\mathcal{L}(P)$ of lower sets with $\operatorname{Ord}(P, \partial I)$ via the lattice anti-homomorphism

$$
\mathcal{L}(P) \rightarrow \operatorname{Ord}(P, \partial I): \alpha \mapsto \Theta_{\alpha}
$$

Now we can state

Stone's Theorem. For every finite poset $P$ and every finite distributive lattice $L$ there are natural isomorphisms

$$
\begin{aligned}
& P \rightarrow \operatorname{Hom}^{*}(\mathcal{L}(P), \partial I): p \mapsto \Theta^{p} \\
& L \rightarrow \mathcal{L}\left(\operatorname{Hom}^{*}(L, \partial I)\right): \alpha \mapsto\{h: h(\alpha)=0\}
\end{aligned}
$$


This version of Stone's theorem is a refinement of what is usually called the Stone Representation Theorem. The category of finite sets can be identified with a subcategory of the category of posets (take the order relation to be the trivial order relation of equality) The category of Boolean algebras is a subcategory of the category of distributive lattices. For the poset $P$ with the trivial order relation the lattice $\mathcal{L}(P)$ of lower sets is the Boolean algebra $2^{P}$ of all subsets of $P$. The Stone Representation Theorem restricts to these subcategories. It asserts that every finite Boolean algebra is isomorphic to the Boolean algebra of subsets of a set $P$.

Associated to every finite poset $P$ there is a natural simplicial complex $K(P)$ whose vertices are the elements of $P$ and whose simplices are the chains (i.e. totally ordered subsets) of $P$. The complex $K(P)$ is well known in combinatorics and algebraic topology [7], [19]. Not every simplicial complex is isomorphic to some $K(P)$ (for example, the boundary of a triangle). However, the barycentric subdivision $\operatorname{sd}(K)$ of any complex $K$ is $K(P)$ in a natural way: the vertices of $\operatorname{sd}(K)$ are the simplices of $K$ and are ordered by inclusion.

The geometric realization $|K(P)|$ of the complex $K(P)$ is the set of all functions $\xi: P \rightarrow I$ such that

$$
\sum_{p \in P} \xi(p)=1, \quad \operatorname{supp}(\xi) \in K(P)
$$

This means that the set of all $p \in P$ with $\xi(p) \neq 0$ is a chain, i.e. a simplex of $K(P)$. For each $p \in P$ let $\Pi_{p}:|K(P)| \rightarrow I$ be the corresponding barycentric coordinate function defined by

$$
\Pi_{p}(\xi)=\xi(p) .
$$

The functions $\Pi_{p}$ form a partition of unity on $|K(P)|$.

The natural embedding of the set of vertices into the geometric realization is given by

$$
P \rightarrow|K(P)|: p \mapsto \delta_{p}
$$

where $\delta_{p}(q)=1$ if $p=q$ and $\delta_{p}(q)=0$ if $p \neq q$. Using this identification we extend the pairing $\Theta: P \times \mathcal{L}(P) \rightarrow \partial I$ to

$$
\Theta:|K(P)| \times \mathcal{L}(P) \rightarrow I
$$

where

$$
\Theta(\xi, \alpha)=\sum_{p \notin \alpha} \xi(p) .
$$

As before we use the notation

$$
\Theta(\xi, \alpha)=\Theta^{\xi}(\alpha)=\Theta_{\alpha}(\xi)
$$

The map

$$
|K(P)| \rightarrow I^{\ell} \subset \mathbb{R}^{\ell}: \xi \mapsto \Theta^{\xi}
$$

restricts to an order preserving map $P \rightarrow \partial I^{\ell}$ and to an affine map on each simplex. Here $\ell$ is the cardinality of $\mathcal{L}(P)$. 
Theorem 2.1. The map $|K(P)| \rightarrow I^{\ell}: \xi \mapsto \Theta^{\xi}$ is an embedding. Its image is the set $\operatorname{Hom}^{*}(\mathcal{L}(P), I)$ which is therefore a polyhedron in the unit cube $I^{\ell} \subset \mathbb{R}^{\ell}$. The vertices of this polyhedron are the elements of the Stone space $\operatorname{Hom}^{*}(\mathcal{L}(P), \partial I)$.

Proof. We show that $\Theta^{\xi}$ is a lattice anti-homomorphism, that is

$$
\Theta^{\xi}(\alpha \cup \beta)=\Theta^{\xi}(\alpha) \wedge \Theta^{\xi}(\beta), \quad \Theta^{\xi}(\alpha \cap \beta)=\Theta^{\xi}(\alpha) \vee \Theta^{\xi}(\beta)
$$

Let $c=\left\{p_{0} \preccurlyeq p_{1} \preccurlyeq \cdots \preccurlyeq p_{k}\right\}$ be the support of $\xi$ and note that

$$
c \cap \alpha=\left\{p_{0}, \ldots, p_{r}\right\}, \quad c \cap \beta=\left\{p_{0}, \ldots, p_{s}\right\}
$$

for some $r$ and $s$. Without loss of generality assume $r \leq s$. Then

$$
\begin{aligned}
& \Theta^{\xi}(\alpha \cup \beta)=\Theta^{\xi}(\beta)=\sum_{i=s+1}^{k} \xi\left(p_{i}\right)=\Theta^{\xi}(\alpha) \vee \Theta^{\xi}(\beta) \\
& \Theta^{\xi}(\alpha \cap \beta)=\Theta^{\xi}(\alpha)=\sum_{i=r+1}^{k} \xi\left(p_{i}\right)=\Theta^{\xi}(\alpha) \wedge \Theta^{\xi}(\beta) .
\end{aligned}
$$

We show that $\xi \mapsto \Theta^{\xi}$ is one-one. Assume that $\xi \neq \eta$. There exist elements $p \in P$ with $\xi(p) \neq \eta(p)$, choose a macimal one. Let $\alpha$ be the lower set of all $q \in P$ with $p \npreceq q$. Then

$$
\Theta^{\xi}(\alpha)=\sum_{p \preccurlyeq q} \xi(q) \neq \sum_{p \preccurlyeq q} \eta(q)=\Theta^{\eta}(\alpha)
$$

We show that $\xi \mapsto \Theta^{\xi}$ is onto. Choose $h \in \operatorname{Hom}^{*}(\mathcal{L}(P), I)$. We construct $\xi \in$ $|K(P)|$ such that $\Theta^{\xi}=h$. For any element $p \in P$ there are lower sets $\alpha, \beta \in \mathcal{L}(P)$ such that $\beta=\alpha \cup\{p\}$ and we define

$$
\xi(p)=h(\alpha)-h(\beta)
$$

The right hand side is independent of the choice of $\alpha$ and $\beta$. To prove this assume that $\beta_{1} \backslash \alpha_{1}=\beta_{2} \backslash \alpha_{2}=\{p\}$. Assume w.l.o.g. that $\beta_{1} \subset \beta_{2}$ so that $\beta_{2}=\beta_{1} \cup \alpha_{2}$ and $\alpha_{1}=\beta_{1} \cap \alpha_{2}$. Then

$$
\begin{aligned}
h\left(\beta_{2}\right)-h\left(\alpha_{2}\right) & =h\left(\beta_{1} \cup \alpha_{2}\right)-h\left(\alpha_{2}\right) \\
& =h\left(\beta_{1}\right) \wedge h\left(\alpha_{2}\right)-h\left(\alpha_{2}\right) \\
& =h\left(\beta_{1}\right)-h\left(\beta_{1}\right) \vee h\left(\alpha_{2}\right) \\
& =h\left(\beta_{1}\right)-h\left(\beta_{1} \cap \alpha_{2}\right) \\
& =h\left(\beta_{1}\right)-h\left(\alpha_{1}\right)
\end{aligned}
$$

(This argument is analogous to the proof of Lemma 1.6.) To prove that the support of $\xi$ is a chain in $P$ we choose any two incomparable elements $p, q \in P$. Then there exists a lower set $\alpha \in \mathcal{L}(P)$ which contains both $p$ and $q$ as maximal elements and hence

$$
\xi(p)=h(\alpha \backslash\{p\})-h(\alpha), \quad \xi(q)=h(\alpha \backslash\{q\})-h(\alpha) .
$$


Since $h$ is a lattice anti-homomorphism we have $h(\alpha)=h(\alpha \backslash\{p\}) \wedge h(\alpha \backslash\{q\})$ and this implies

$$
\xi(p) \wedge \xi(q)=0
$$

We conclude that either $\xi(p)=0$ or $\xi(q)=0$ for any two incomparable elements $p, q \in P$. Moreover $\sum_{p \in P} \xi(p)=h(\emptyset)-h(P)=0$ and therefore $\xi \in|K(P)|$. It follows directly from the definition of $\xi$ that $\Theta^{\xi}=h$.

Remark. The following argument is suggestive but awkward to make precise:

$$
\begin{aligned}
\operatorname{Hom}^{*}(\mathcal{L}(P), I) & =\operatorname{Hom}^{*}(\mathcal{L}(P), \mathcal{L}(I)) \\
& =\operatorname{Ord}^{*}(I, P) \\
& =K(P)
\end{aligned}
$$

The equation $I=\mathcal{L}(I)$ used in the first line holds because $I$ is totally ordered. The second line is Stone's theorem which asserts that lattice anti-homomorphisms from $\mathcal{L}(P)$ to $\mathcal{L}(Q)$ correspond to order reversing maps from $Q$ to $P$. The last line is justified by an analysis of what an order reversing map from $I$ to $P$ must look like.

The rectalinear coordinates $\Theta_{\alpha}:|K(P)| \rightarrow I$ and the barycentric coordinates $\Pi_{p}:|K(P)| \rightarrow I$ are related by

$$
\Theta_{\alpha}=\sum_{p \notin \alpha} \Pi_{p}
$$

The rectalinear coordinate $\Theta_{\alpha}$ can be interpreted geometrically as follows. Since $\alpha$ is a lower set, the complex $K(P)$ is the join of the subcomplexes $K(\alpha)$ and $K(P \backslash \alpha)$ and $\Theta_{\alpha}$ is the join-parameter. This means that any $\xi \in|K(P)|$ may be written uniquely in the form

$$
\xi=(1-t) \xi_{0}+t \xi_{1}
$$

where $\xi_{0} \in|K(\alpha)|, \xi_{1} \in|K(P \backslash \alpha)|$ and $0 \leq t \leq 1$. The value of $t$ is the number $\Theta_{\alpha}(\xi)$.

We construct a dynamical system $f_{P}$ on $M_{P}=|K(P)|$ which we call a standard system on $|K(P)|$. Its attractors are precisely the subcomplexes $|K(\alpha)|$ for $\alpha \in$ $\mathcal{L}(P)$ so that the map

$$
\mathcal{L}(P) \rightarrow \mathcal{A}\left(M_{P}, f_{P}\right): \alpha \mapsto A_{\alpha}=|K(\alpha)|
$$

is a lattice isomorphism. For this we choose real numbers $\rho_{p} \in \mathbb{R}$ satisfying the condition

$$
p \preccurlyeq q, \quad p \neq q \quad \Longrightarrow \quad \rho_{p}<\rho_{q}
$$

but otherwise arbitrary. Let the homeomorphism $f_{P}:|K(P)| \rightarrow|K(P)|$ be the time-one map of the flow generated by the differential equations

$$
\dot{\xi}_{p}=\sum_{q \in P}\left(\rho_{q}-\rho_{p}\right) \xi_{q} \xi_{p}
$$


(Here we write $\xi_{p}$ instead of $\xi(p)$.) This flow is naturally induced by a linear flow on the projective space of $\mathbb{R}^{P}$ with eigenvalues $\rho_{p}$. The map which sends $\xi \in|K(P)|$ to the line $\mathbb{R} \xi \subset \mathbb{R}^{P}$ intertwines the two flows. Note that

$$
\begin{aligned}
\dot{\Theta}_{\alpha} & =\sum_{p \notin \alpha} \dot{\xi}_{p} \\
& =\sum_{p \notin \alpha} \sum_{q \in P}\left(\rho_{q}-\rho_{p}\right) \xi_{q} \xi_{p} \\
& =\sum_{p \notin \alpha} \sum_{q \in \alpha}\left(\rho_{q}-\rho_{p}\right) \xi_{q} \xi_{p} \\
& \leq 0 .
\end{aligned}
$$

The penultimate step follows from the skew-symmetry of the summand in the indices $p$ and $q$. Each simplex of $|K(P)|$ is invariant under the flow since $\xi_{p}$ factors out of the expression for $\dot{\xi}_{p}$. Choose $\xi \in|K(P)|$. Then the set of $p \in P$ with $\xi_{p} \neq 0$ is a chain in $P$. Hence the monotonicity condition on the $\rho_{p}$ implies that $\dot{\Theta}_{\alpha} \leq 0$ with equality holding if and only if $\xi \in|K(\alpha)| \cup|K(P \backslash \alpha)|$. It follows that the attractors for $f$ in $M$ are precisely the subcomplexes $|K(\alpha)|$ with $\alpha \in \mathcal{L}(P)$. The associated basic sets are the vertices of $K(P)$ and the order of $P$ is the smallest partial order extending the relation $p \preccurlyeq_{0} q$ if and only if there is a connecting orbit from $q$ to $p$.

\section{$\S 3$ LYAPUNOV MAPS}

Continue the notation of the previous sections. A Lyapunov function for $(M, f)$ is a continuous function $\theta: M \rightarrow I$ which is nonincreasing along orbits

$$
\theta\left(f^{t}(x)\right) \leq \theta(x) \quad \text { for } x \in M \text { and } t>0
$$

and strictly decreasing precisely where $\theta(x) \neq 0,1$; i.e. for $t>0$ we have

$$
\theta\left(f^{t}(x)\right)<\theta(x) \quad \Longleftrightarrow \quad 0<\theta(x)<1
$$

It follows that

$$
A=\theta^{-1}(0), \quad A^{*}=\theta^{-1}(1)
$$

is an attractor-repeller pair. A Lyapunov function with $A=\theta^{-1}(0)$ is said to define $A$. In Proposition 1.4 we showed how to construct a Lyapunov function defining a given attractor $A \in \mathcal{A}(M, f)$. If $\theta_{i}: M \rightarrow I$ is a Lyapunov function for $(M, f)$ defining the attractor $A_{i}$ for $i=1,2$ then $\theta_{1} \wedge \theta_{2}=\min \left(\theta_{1}, \theta_{2}\right)$ is a Lyapunov function defining $A_{1} \cup A_{2}$ and $\theta_{1} \vee \theta_{2}=\max \left(\theta_{1}, \theta_{2}\right)$ is a Lyapunov function defining $A_{1} \cap A_{2}$. Thus we have shown 
Proposition 3.1. The space $\mathcal{F}(M, f)$ of Lyapunov functions for $(M, f)$ is a distributive lattice and the map

$$
\mathcal{F}(M, f) \rightarrow \mathcal{A}(M, f): \theta \mapsto \theta^{-1}(0)
$$

is a lattice anti-epimorphism.

Strong conjecture. This map admits a section, i.e. there exists a lattice antihomo-morphism $\mathcal{A}(M, f) \rightarrow \mathcal{F}(M, f): A \mapsto \theta_{A}$ such that $\theta_{A}$ defines $A$ for every $A \in \mathcal{A}(M, f)$.

Recall that a compact set $N \subset M$ is called an attracting neighborhood for $(M, f)$ if $f(N) \subset \operatorname{int}(N)$. The set $\mathcal{N}(M, f)$ of compact attracting neighborhoods forms a lattice. There is a natural lattice epimorphism

$$
\mathcal{N}(M, f) \rightarrow \mathcal{A}(M, f): N \mapsto \bigcap_{t \geq 0} f^{t}(N)
$$

Weak conjecture. This map admits a section, i.e. there exists a lattice homomorphism $\mathcal{A}(M, f) \rightarrow \mathcal{N}(M, f): A \mapsto N_{A}$ such that $N_{A}$ is an attracting neighborhood of $A$.

The strong conjecture implies the weak conjecture since we may take $N_{A}=$ $\theta_{A}{ }^{-1}\left(\left[0, \frac{1}{2}\right]\right)$. In case the lattice $\mathcal{A}(M, f)$ is finite, the strong conjecture is a corollary of our main theorem.

Recall that a lattice homomorphism $\mathcal{L}(P) \rightarrow \mathcal{A}(M, f): \alpha \mapsto A_{\alpha}$ from the lattice of lower sets of a finite poset $P$ to the lattice of attractors is called an attractor network. A lattice anti-homomorphism $\mathcal{L}(P) \rightarrow \mathcal{F}(M, f): \alpha \mapsto \theta_{\alpha}$ is called a Lyapunov network. This is a collection of Lyapunov functions $\left\{\theta_{\alpha}\right\}_{\alpha}$ satisfying

$$
\theta_{\alpha \cup \beta}=\theta_{\alpha} \wedge \theta_{\beta}, \quad \theta_{\alpha \cap \beta}=\theta_{\alpha} \vee \theta_{\beta}, \quad \theta_{\emptyset}=1, \quad \theta_{P}=0 .
$$

If in addition we have that

$$
A_{\alpha}=\theta_{\alpha}^{-1}(0)
$$

we say that the Lyapunov network $\left\{\theta_{\alpha}\right\}_{\alpha}$ defines the attractor network $\left\{A_{\alpha}\right\}_{\alpha}$. A lattice homomorphism $\mathcal{L}(P) \rightarrow \mathcal{N}(M, f): \alpha \mapsto N_{\alpha}$ is called a neighborhood network. This is a collection $\left\{N_{\alpha}\right\}_{\alpha}$ of compact attracting neighborhoods such that

$$
N_{\alpha \cup \beta}=N_{\alpha} \cup N_{\beta}, \quad N_{\alpha \cap \beta}=N_{\alpha} \cap N_{\beta}, \quad N_{\emptyset}=\emptyset, \quad N_{P}=M .
$$

If in addition we have that

$$
A_{\alpha}=\bigcap_{t \geq 0} f^{t}\left(N_{\alpha}\right)
$$

we say that the neighborhood network $\left\{N_{\alpha}\right\}_{\alpha}$ defines the attractor network $\left\{A_{\alpha}\right\}_{\alpha}$. 
A Lyapunov map for $(M, f)$ is a continuous function

$$
\psi: M \rightarrow|K(P)|
$$

such that

$$
\theta_{\alpha}=\Theta_{\alpha} \circ \psi \in \mathcal{F}(M, f)
$$

is a Lyapunov function for every $\alpha \in \mathcal{L}(P)$. A Lyapunov map determines an attractor network $\left\{A_{\alpha}\right\}_{\alpha \in \mathcal{L}(P)}$ via the equations $A_{\alpha}=\theta_{\alpha}^{-1}(0)$ : we say that the Lyapunov map defines this attractor network. Here is our main theorem.

Theorem 3.2. Any attractor network is defined by a Lyapunov map $\psi$.

A Lyapunov map is uniquely determined by the collection of functions

$$
\pi_{p}=\Pi_{p} \circ \psi: M \rightarrow I, \quad p \in P
$$

called a Lyapunov partition of unity. The function $\pi_{p}$ and $\theta_{\alpha}$ are related by

$$
\theta_{\alpha}=\sum_{p \notin \alpha} \pi_{p}
$$

If $\psi: M \rightarrow|K(P)|$ is a Lyapunov map then the collection of Lyapunov functions $\left\{\theta_{\alpha}\right\}_{\alpha \in \mathcal{L}(P)}$ determines a lattice anti-homomorphism $\mathcal{L}(P) \rightarrow \mathcal{F}(M, f)$. Conversely, every lattice anti-homomorphism

$$
\mathcal{L}(P) \rightarrow \mathcal{F}(M, f): \alpha \mapsto \theta_{\alpha}
$$

defines a map

$$
M \times \mathcal{L}(P) \rightarrow I:(x, \alpha) \mapsto \theta_{\alpha}(x)
$$

whose restriction to $\{x\} \times \mathcal{L}(P)$ is a lattice anti-homomorphism which by Proposition 2.1 corresponds to a point $\psi(x) \in|K(P)|$. Thus there is a one-to-one correspondence between Lyapunov partitions of unity $\left\{\pi_{p}\right\}_{p}$ and Lyapunov networks $\left\{\theta_{\alpha}\right\}_{\alpha}$. This correspondence resembles the relation between partially ordered sets and distributive lattices. This discussion shows that a Lyapunov map and a Lyapunov network determine one another uniquely so we have the following reformulation of Theorem 3.2:

Theorem 3.3. Any attractor network can be defined by some Lyapunov network. In other words, any lattice homomorphism $\mathcal{L}(P) \rightarrow \mathcal{A}(M, f)$ lifts to a lattice antihomomorphism $\mathcal{L}(P) \rightarrow \mathcal{F}(M, f)$.

It is in this form that we prove our main theorem. See $\S 5$. Finally, just as the strong conjecture implies the weak conjecture so does Theorem 3.3 imply the following

Corollary 3.4 (Franzosa [8]). Any attractor network can be defined by some neighborhood network. In other words, any lattice homomorphism $\mathcal{L}(P) \rightarrow \mathcal{A}(M, f)$ lifts to a lattice homomorphism $\mathcal{L}(P) \rightarrow \mathcal{N}(M, f)$. 


\section{$\S 4$ NeIGHBORHoOd NETWORKS}

In this section we construct a neighborhood network with a certain additional property and use them to construct a Lyapunov network. Throughout $\left\{A_{\alpha}\right\}_{\alpha}$ denotes an attactor network for $(M, f)$ indexed by the lower sets $\alpha \in \mathcal{L}(P)$ of a poset $P$. We assume that $A_{\alpha} \neq A_{\beta}$ for $\alpha \neq \beta$.

Proposition 4.1. Let $\left\{N_{\alpha}\right\}_{\alpha}$ be a neighborhood network defining the attractor network $\left\{A_{\alpha}\right\}_{\alpha}$. For any $\alpha, \beta \in \mathcal{L}(P)$ define

$$
C_{\beta \backslash \alpha}=N_{\beta} \backslash N_{\alpha}
$$

and for $p \in P$ let $C_{p}=C_{\{p\}}$. The set $C_{\beta \backslash \alpha}$ is well defined. The sets $C_{p}$ partition $M$ and the various sets $N_{\alpha}$ :

$$
\begin{gathered}
M=\bigcup_{p \in P} C_{p}, \\
C_{p} \cap C_{q}=\emptyset \quad \text { for } p, q \in P \text { and } p \neq q, \\
N_{\alpha}=\bigcup_{p \in \alpha} C_{p}, \\
C_{\beta \backslash \alpha}=\bigcup_{p \in \beta \backslash \alpha} C_{p} .
\end{gathered}
$$

Proof. To prove this assume that $\beta_{1} \backslash \alpha_{1}=\beta_{2} \backslash \alpha_{2}$. Assume w.l.o.g. that $\beta_{1} \subset \beta_{2}$ and $\alpha_{1} \subset \alpha_{2}$ so that $\beta_{2}=\beta_{1} \cup \alpha_{2}$ and $\alpha_{1}=\beta_{1} \cap \alpha_{2}$. Then

$$
\begin{aligned}
N_{\beta_{2}} \backslash N_{\alpha_{2}} & =N_{\beta_{1} \cup \alpha_{2}} \backslash N_{\alpha_{2}} \\
& =\left(N_{\beta_{1}} \cup N_{\alpha_{2}}\right) \backslash N_{\alpha_{2}} \\
& =N_{\beta_{1}} \backslash\left(N_{\beta_{1}} \cap N_{\alpha_{2}}\right) \\
& =N_{\beta_{1}} \backslash N_{\beta_{1} \cap \alpha_{2}} \\
& =N_{\beta_{1}} \backslash N_{\alpha_{1}}
\end{aligned}
$$

(This argument is analogous to the proof of Lemma 1.6.)

We call the sets $C_{p}$ the cells of the neighborhood network $\left\{N_{\alpha}\right\}_{\alpha}$ The sets $C_{p}$ need be neither open nor closed. Conditions (4-1) and (4-2) guarantee that the map $\alpha \mapsto N_{\alpha}$ defined by (4-3) is a homomorphism of lattices. It is easy to see that the cells must also satisfy the properties

$$
\begin{gathered}
\operatorname{cl}\left(C_{p}\right) \cap A_{\alpha}=\emptyset \quad \text { for } p \notin \alpha . \\
\Lambda_{p} \subset \operatorname{int}\left(C_{p}\right)
\end{gathered}
$$

We say that the neighborhood network $\left\{N_{\alpha}\right\}_{\alpha}$ has the separation property if incomparable cells have disjoint closures:

$$
\operatorname{cl}\left(C_{p}\right) \cap \operatorname{cl}\left(C_{q}\right)=\emptyset \quad \text { for } p \nprec q \text { and } q \npreceq p .
$$


Theorem 4.2. There is a neighborhood network with the separation property defining the attractor network $\left\{A_{\alpha}\right\}_{\alpha}$.

Proof. Suppose by induction that the sets $C_{p}$ have been constructed for all $p \in \gamma$ for some lower set $\gamma \in \mathcal{L}(P)$, that they satisfy conditions (4-2), (4-4), (4-5), (4-6), and that $N_{\alpha}$ as defined in (4-3) is a compact attracting neighborhood of $A_{\alpha}$ for every lower set $\alpha \subset \gamma$. Choose $r \in P \backslash \gamma$ such that $\delta=\gamma \cup\{r\}$ is a lower set. We shall define $C_{r}$ and verify that it satisfies the induction hypotheses.

Consider the lower set

$$
\rho=\{p \in P: p \preccurlyeq r\} \quad \subset \quad \delta .
$$

We claim that there is a compact attracting neighborhood $W$ of $A_{\rho}$ such that

(i) $W \cap \operatorname{cl}\left(C_{p}\right)=\emptyset$ for $p \in \gamma \backslash \rho$.

(ii) $\operatorname{cl}\left(W \backslash N_{\gamma}\right) \cap A_{\alpha}=\emptyset$ for every lower set $\alpha \in \mathcal{L}(P)$ with $r \notin \alpha$.

For this we first show that that (i) and (ii) are satisfied with $W$ replaced by $A_{\rho}$. In the case of (i) this follows from condition (4-4). To prove this for (ii) assume otherwise that

$$
x \in \operatorname{cl}\left(A_{\rho} \backslash N_{\gamma}\right) \cap A_{\alpha} .
$$

and $r \notin \alpha$. Then $x \in A_{\rho} \cap A_{\alpha}=A_{\rho \cap \alpha}$ and since $\rho \cap \alpha \subset \gamma$ we obtain $x \in$ $\operatorname{int}\left(N_{\gamma}\right)$, a contradiction. So it follows that conditions (i) and (ii) are satisfied for $W=f^{t}(\operatorname{cl}(U))$ where $U$ is any attracting neighborhood of $A_{\rho}$ and the number $t$ is sufficiently large. Let

$$
C_{r}=W \backslash N_{\gamma} .
$$

We must verify the induction hypotheses. Conditions (4-2), (4-4), (4-5), (4-6) are obvious; we need only prove that $N_{\beta}$ is a compact attracting neighborhood of $A_{\beta}$ for any lower set $\beta \subset \delta$ with $r \in \beta$. Any such set must contain $\rho$ and we consider first the case $\beta=\rho$. It follows from (i) that the cells in $N_{\gamma}$ which do not lie in $N_{\rho \backslash\{r\}}$ do not intersect $W$ and hence

$$
N_{\rho}=\bigcup_{p \in \rho} C_{p}=C_{r} \cup N_{\rho \backslash\{r\}}=W \cup N_{\rho \backslash\{r\}} .
$$

Both sets $W$ and $N_{\rho \backslash\{r\}}$ are compact attracting neighborhoods and hence so is their union $N_{\rho}$. Now any lower set $\beta \subset \delta$ with $r \in \beta$ can be written as the union

$$
\beta=\alpha \cup \rho
$$

where $\alpha=\beta \backslash\{r\}=\beta \cap \gamma$. Let $N_{\beta}$ be defined by (4-3). Then

$$
N_{\beta}=\bigcup_{p \in \beta} C_{p}=N_{\alpha} \cup N_{\rho}
$$

and $N_{\alpha}$ is a compact attracting neighborhood of $A_{\alpha}$ by induction hypothesis. Hence $N_{\beta}$ is a compact attracting neighborhood of $A_{\beta}$. This finishes the induction and the proof of the theorem. 
Corollary 4.3. Theorem 3.3 holds in the continuous time case: Every attractor network can be defined by a Lyapunov network

Proof. Since $N_{\alpha}$ is an attracting neighborhood it follows that $f^{t}\left(N_{\alpha}\right) \subset \operatorname{int}\left(N_{\alpha}\right)$ for every $t>0$. This means that every orbit of the flow which is not in $A_{\alpha} \cup A_{\alpha}^{*}$ intesects the set $\partial N_{\alpha}$ in precisely one point. Therefore the function $t_{\alpha}: M \rightarrow[-\infty, \infty]$ defined by

$$
t_{\alpha}\left(f^{t}(x)\right)=-t
$$

for $x \in \partial N_{\alpha}$ with $t_{\alpha}\left(A_{\alpha}\right)=-\infty$ and $t_{\alpha}\left(A_{\alpha}^{*}\right)=+\infty$ is continuous. The equations

$$
t_{\alpha \cup \beta}=t_{\alpha} \wedge t_{\beta}, \quad t_{\alpha \cap \beta}=t_{\alpha} \vee t_{\beta}
$$

are easily proved. For example, for $y \in N_{\alpha \cap \beta}=N_{\alpha} \cap N_{\beta}$ the backwards orbit of $y$ leaves one of the sets first, say $N_{\alpha}$, and then leaves the other $N_{\beta}$. It exits $N_{\alpha}$ at the same instant that it exits $N_{\alpha \cap \beta}=N_{\alpha} \cap N_{\beta}$ so that $t_{\alpha \cap \beta}(y)=t_{\alpha}(y) \geq t_{\beta}(y)$ (remember the minus sign) which shows that $t_{\alpha \cap \beta}=t_{\alpha} \wedge t_{\beta}$. Now define $\theta_{\alpha}$ by composing with an order preserving isomorphism $\phi:[-\infty, \infty] \rightarrow[0,1]$.

Remark 4.4. The Lyapunov function $\theta_{\alpha}$ are level preserving meaning that there are flows $g_{\alpha}^{t}: I \rightarrow I$ such that

$$
g_{\alpha}^{t} \circ \theta_{\alpha}=\theta_{\alpha} \circ f^{t}
$$

To prove Theorem 3.3 in the discrete time case we reduce it to the continuous time case just proved. Assume $f: M \rightarrow M$ is a homeomorphism and denote the suspension by

$$
\Sigma=\Sigma_{f} M=M \times \mathbb{R} / \equiv
$$

where $(x, s+1) \equiv(f(x), s)$. Note that $\Sigma$ is a second countable compact Hausdorff space and is therefore metrizable. Denote by $\phi^{t}: \Sigma \rightarrow \Sigma$ the flow defined by $\phi^{t}(x, s)=(x, s+t)$. Now Theorem 3.3 in the discrete time case follows from the continuous time case and the following two Lemmata.

Lemma 4.5. If $A \subset M$ is an attractor for $f$ then $\Sigma A \subset \Sigma$ is an attractor for $\phi$. The map

$$
\mathcal{A}(M, f) \rightarrow \mathcal{A}(\Sigma, \phi): A \mapsto \Sigma A
$$

is a lattice homomorphism.

Proof. If $A$ is an attractor for $f$ then $\left(\Sigma A, \Sigma A^{*}\right)$ is an attractor-repeller pair for $\phi$. So the result follows from Proposition 1.4.

Lemma 4.6. If $\theta: \Sigma \rightarrow \mathbb{R}$ is a Lyapunov function for $\phi$ then

$$
\theta \circ \iota: M \rightarrow \mathbb{R}
$$

is a Lyapunov function for $f$. Here $\iota: M \rightarrow \Sigma$ is the inclusion $\iota(x)=(x, 0)$. The map

$$
\mathcal{F}(\Sigma, \phi) \rightarrow \mathcal{F}(M, f): \theta \mapsto \theta \circ \iota
$$

is a lattice homomorphism. 


\section{$\S 5$ Piecewise SMooth Neighborhood Networks}

Throughout this section we assume that $M$ is a compact smooth $n$-dimensional manifold. A subset $N \subset M$ is called a PS-domain (piecewise smooth domain) if each point in $N$ has a neighborhood which is diffeomorphic to an open set in the nonnegative $2^{n}$-tant $[0, \infty)^{n} \subset \mathbb{R}^{n}$. Note that any such PS-domain is stratified: A point $x \in N$ lies in the $k$-dimensional stratum if it admits a neighborhood $U$ such that $(U, x)$ is diffeomorphic to $\left(\mathbb{R}^{k} \times[0, \infty)^{n-k}, 0\right)$. By definition a PS-domain is the closure of its $n$-dimensional stratum. Two PS-domains $N_{1}, N_{2} \subset M$ intersect transversally if every stratum of $N_{1}$ intersects every stratum of $N_{2}$ transversally. If two PS-domains intersect transversally then their intersection $N_{1} \cap N_{2}$ is a PSdomain. Note that this does not hold for unions. A PS-codomain is the closure of the complement of a PS-domain.

Theorem 5.1. Let $\left\{A_{\alpha}\right\}_{\alpha \in \mathcal{L}(P)}$ be an attractor network for a smooth dynamical system $f$ on a smooth manifold $M$. Then there is a neighborhood network $\left\{N_{\alpha}\right\}_{\alpha \in \mathcal{L}(P)}$ defining the given attractor network such that each $N_{\alpha}$ is a PS-codomain and each closed cell $\mathrm{cl}\left(C_{p}\right)$ is a PS-domain.

Proof. Let $\gamma$ be a lower set. Suppose that the cells $C_{p}$ have been constructed for $p \in \gamma$ such that the induction hypotheses in the proof of Theorem 4.2 are satisfied and the statement of Theorem 5.1 holds for every lower set $\alpha \subset \gamma$. Choose $r \in P \backslash \gamma$ such that $\delta=\gamma \cup\{r\}$ is a lower set. To construct the cell $C_{r}$ we shall proceed as in the proof of Theorem 4.2.

Let $\rho$ be the smallest lower set containing $r$. By Proposition 1.4 there exists a smooth Lyapunov function $\theta: M \rightarrow[0,1]$ for the attractor $A_{\rho}$. By Sard's theorem almost every number $t \in[0,1]$ is a regular value for the restriction of $\theta$ to every stratum in $N_{\alpha}$ for every lower set $\alpha \subset \gamma$. Choose $t>0$ to be such a regular value and sufficiently small. Then the submanifold with boundary

$$
W=\theta^{-1}([0, t])
$$

intersects the $P S$-codomain $N_{\alpha}$ transversally for every lower set $\alpha \subset \gamma$. Hence the union

$$
N_{\alpha \cup\{r\}}=N_{\alpha} \cup W
$$

is a $P S$-codomain for every lower set $\alpha \subset \gamma$ and

$$
\operatorname{cl}\left(C_{r}\right)=\operatorname{cl}\left(W \backslash N_{\gamma}\right)=W \cap \operatorname{cl}\left(M \backslash N_{\gamma}\right)
$$

is a $P S$-domain. It follows as in Theorem 4.2 that the induction hypotheses are satisfied with $\gamma$ replaced by $\delta$.

Corollary 5.2. The theorem continues to hold with the word codomain replaced by domain. (The sets $N_{\alpha}$ and $\operatorname{cl}\left(C_{p}\right)$ are all PS-domains.)

Proof. Let $\left\{N_{\alpha}^{*}\right\}_{\alpha \in \mathcal{L}(P)}$ be a neighborhood network for the reversed dynamical system $f^{-1}$ defining the attractor network $\left\{A_{\alpha}^{*}\right\}_{\alpha \in \mathcal{L}(P)}$ and satisfying the requirements of Theorem 5.1. Let

$$
N_{\alpha}=\operatorname{cl}\left(M \backslash N_{\alpha}^{*}\right)=M \backslash \operatorname{int}\left(N_{\alpha}^{*}\right)
$$


We will prove that if $\alpha \subset \beta$ then

$$
\operatorname{cl}\left(N_{\beta} \backslash N_{\alpha}\right)=\operatorname{cl}\left(\operatorname{int}\left(N_{\beta}\right) \cap \operatorname{int}\left(N_{\alpha}^{*}\right)\right)=\operatorname{cl}\left(N_{\alpha}^{*} \backslash N_{\beta}^{*}\right)
$$

To see this note first that the complement of $N_{\alpha}$ is the interior of $N_{\alpha}^{*}$ and hence

$$
\operatorname{cl}\left(N_{\beta} \backslash N_{\alpha}\right)=\operatorname{cl}\left(N_{\beta} \cap \operatorname{int}\left(N_{\alpha}^{*}\right)\right) .
$$

Since $N_{\beta}=\operatorname{cl}\left(\operatorname{int}\left(N_{\beta}\right)\right)$ it follows that

$$
\operatorname{cl}\left(N_{\beta} \cap \operatorname{int}\left(N_{\alpha}^{*}\right)\right)=\operatorname{cl}\left(\operatorname{int}\left(N_{\beta}\right) \cap \operatorname{int}\left(N_{\alpha}^{*}\right)\right)
$$

and this proves the first identity in (5-1). The second identity follows by similar arguments. Thus we have proved (5-1) and hence $\operatorname{cl}\left(C_{p}\right)$ is a PS-domain for every $p \in P$.

\section{$\S 6$ HOMOLOGY BRAIDS}

Let $\left\{A_{\alpha}\right\}_{\alpha \in \mathcal{L}(P)}$ be an attractor network for a smooth dynamical system $f^{t}$ : $M \rightarrow M$ on a compact smooth manifold $M$. Let $\left\{N_{\alpha}\right\}_{\alpha \in \mathcal{L}(P)}$ be a piecewise smooth neighborhood network defining this attractor network as in Theorem 5.1. By lemma 1.7 the set

$$
\Lambda_{\beta \backslash \alpha}=A_{\beta} \cap A_{\alpha}^{*}
$$

depends only on the difference $\beta \backslash \alpha$ of the pair of lower sets $\alpha \subset \beta$. In the terminology of [15] $\left(N_{\beta}, N_{\alpha}\right)$ forms an index pair for the isolated invariant set $\Lambda_{\beta \backslash \alpha}$. The upshot of this is that the index pair can be used to define topological invariants of the isolated invariant set which are independent of the choice of the index pair.

Since the pair $\left(N_{\beta}, N_{\alpha}\right)$ can be triangulated the homology $H\left(N_{\beta}, N_{\alpha}\right)$ is finitely generated for any two lower sets $\alpha \subset \beta$. By the excision theorem, the homology group $H\left(N_{\beta}, N_{\alpha}\right)$ depends only on the set-theoretic difference $\beta \backslash \alpha$ up to canonical isomorphism. In the continuous-time case the homotopy type of the space $N_{\beta} / N_{\alpha}$ and hence the homology $H\left(N_{\beta}, N_{\alpha}\right)$ is independent of the choice of the index pair defining it and hence of the neighborhood network. The homotopy type of $N_{\beta} / N_{\alpha}$ is called the Conley index of the isolated invariant set $\Lambda_{\beta \backslash \alpha}$; its homology $H\left(N_{\beta}, N_{\alpha}\right)$ is called the homological Conley index. The arguments which justify this generalize to the discrete-time case but yield shape invariants rather than homotopy invariants. We recall how this works.

There is an endomorphism $H(f): H\left(N_{\beta}, N_{\alpha}\right) \rightarrow H\left(N_{\beta}, N_{\alpha}\right)$ induced by the diffeomorphism $f$ on homology. The direct limit $\mathcal{H}\left(N_{\beta}, N_{\alpha}\right)=\lim \left(H\left(N_{\beta}, N_{\alpha}\right), H(f)\right)$ of the direct system

$$
H\left(N_{\beta}, N_{\alpha}\right) \stackrel{H(f)}{\longrightarrow} H\left(N_{\beta}, N_{\alpha}\right) \stackrel{H(f)}{\longrightarrow} \cdots
$$

is the module of equivalence classes of pairs $[a, k]$ where $a \in H\left(N_{\beta}, N_{\alpha}\right)$ and $k \in \mathbb{Z}$ under the equivalence relation generated by

$$
[a, k] \equiv[H(f) a, k+1]
$$


The induced shift automorphism is given by

$$
\mathcal{H}(f): \mathcal{H}\left(N_{\beta}, N_{\alpha}\right) \rightarrow \mathcal{H}\left(N_{\beta}, N_{\alpha}\right), \quad[a, k] \mapsto[H(f) a, k]
$$

with the inverse $[a, k] \mapsto[a, k+1]$. If the coefficient ring is a field then this direct limit can be identified with the quotient of $H\left(N_{\beta}, N_{\alpha}\right)$ by the generalized kernel of $H(f)$, that is the kernel of a high power.

Consider the example where $H\left(N_{\beta}, N_{\alpha}\right)=\mathbb{Z}$ and $H(f)$ acts by multiplication with 2 . In this case $\mathcal{H}\left(N_{\beta}, N_{\alpha}\right)$ is the $\mathbb{Z}$-module of rational numbers whose denominator is a power of 2 . This situation occurs when $\Lambda_{\beta \backslash \alpha}$ is the isolated invariant set in a figure-G horseshoe.

If $f$ is the time-one map of a flow then the map $H(f)$ is the identity and so $\mathcal{H}\left(N_{\beta}, N_{\alpha}\right)=H\left(N_{\beta}, N_{\alpha}\right)$. For discrete-time systems the direct limit $\mathcal{H}\left(N_{\beta}, N_{\alpha}\right)$ is the natural analogue of the homological Conley index in the continuous-time case. It corresponds to the shape equivalence class of the inverse system $f: N_{\beta} / N_{\alpha} \rightarrow$ $N_{\beta} / N_{\alpha}$ just as the usual homology groups correspond to the homotopy type of the topological quotient $N_{\beta} / N_{\alpha}$. The direct limit $\mathcal{H}\left(N_{\beta}, N_{\alpha}\right)$ is a shape invariant for the isolated invariant set $\Lambda_{\beta \backslash \alpha}$ and does not depend on the choice of the neighborhood network. For details of the shape index we refer to [15]. We formulate and prove the consequence of this theory which is of interest here:

Proposition 6.1. Let $\left\{N_{\alpha}\right\}_{\alpha \in \mathcal{L}(P)}$ and $\left\{N_{\alpha}^{\prime}\right\}_{\alpha \in \mathcal{L}(P)}$ be two neighborhood networks defining the same attractor network $\left\{A_{\alpha}\right\}_{\alpha \in \mathcal{L}(P)}$. Then there are canonical isomorphisms

$$
\mathcal{H}\left(N_{\beta}, N_{\alpha}\right) \rightarrow \mathcal{H}\left(N_{\beta}^{\prime}, N_{\alpha}^{\prime}\right)
$$

Proof. The map

$$
\phi^{\ell}: N_{\beta} / N_{\alpha} \rightarrow N_{\beta}^{\prime} / N_{\alpha}^{\prime}
$$

defined by

$$
\phi^{\ell}(x)= \begin{cases}f^{\ell}(x) & \text { if } f^{j}(x) \in N_{\beta} \backslash N_{\alpha} \text { for } 0 \leq j \leq \frac{2 \ell}{3} \\ & \text { and } f^{j}(x) \in N_{\beta}^{\prime} \backslash N_{\alpha}^{\prime} \text { for } \frac{\ell}{3} \leq j \leq \ell, \\ & \text { otherwise }\end{cases}
$$

is continuous for sufficiently large $\ell[15]$. Let

$$
\psi^{m}: N_{\beta}^{\prime} / N_{\alpha}^{\prime} \rightarrow N_{\beta} / N_{\alpha}
$$

be defined analogously. Then $\phi^{\ell}$ and $\psi^{m}$ intertwine the induced semidynamical systems (still denoted by $f$ ) on $N_{\beta} / N_{\alpha}$ and $N_{\beta}^{\prime} / N_{\alpha}^{\prime}$ :

$$
\phi^{\ell} \circ f=f \circ \phi^{\ell}, \quad \psi^{m} \circ f=f \circ \psi^{m}, \quad \psi^{m} \circ \phi^{\ell}=f^{\ell+m}, \quad \phi^{\ell} \circ \psi^{m}=f^{\ell+m} .
$$

This shows that the map

$$
\mathcal{H}\left(N_{\beta}, N_{\alpha}\right) \rightarrow \mathcal{H}\left(N_{\beta}^{\prime}, N_{\alpha}^{\prime}\right):[a, k] \mapsto\left[H\left(\phi^{\ell}\right) a, k+\ell\right]
$$

is an isomorphism. 
By Proposition 6.1 the homology

$$
\mathcal{H}\left(\Lambda_{\beta \backslash \alpha}\right)=\mathcal{H}\left(N_{\beta}, N_{\alpha}\right)
$$

is independent of both the choice of the pair $\alpha \subset \beta$ of lower sets giving $\beta \backslash \alpha$ and the neighborhood network $\left\{N_{\alpha}\right\}_{\alpha}$ defining the attractor network $\left\{A_{\alpha}\right\}_{\alpha}$. These homologies determine a collection of homology exact triangles

$$
\mathcal{H}\left(\Lambda_{\beta \backslash \alpha}\right) \stackrel{i}{\rightarrow} \mathcal{H}\left(\Lambda_{\gamma \backslash \alpha}\right) \stackrel{p}{\rightarrow} \mathcal{H}\left(\Lambda_{\gamma \backslash \beta}\right) \stackrel{d}{\rightarrow} \mathcal{H}\left(\Lambda_{\beta \backslash \alpha}\right)
$$

for any three lower sets $\alpha \subset \beta \subset \gamma$. This collection is called the homology braid by Franzosa because they satisfy a certain commutative diagram which looks like a braid. In the continuous time case each exact triangle of the homology braid is the homology exact sequence of a triple; in the discrete time case the homology braid is obtained from the exact sequence of the triple by taking direct limits. The induced shift automorphism

$$
\mathcal{H}\left(\Lambda_{\beta \backslash \alpha}\right) \stackrel{\mathcal{H}(f)}{\longrightarrow} \mathcal{H}\left(\Lambda_{\beta \backslash \alpha}\right)
$$

is the identity in the continuous time case and intertwines the homology exact triangles in the general case. It is our aim in the next section to provide a theory of chain complexes which will yield these homology braids.

\section{$\S 7$ Chain Representations}

Fix a partially ordered set $P$. All modules are over an unspecified ring called the coefficient ring. A $P$-filtered module is a module $X$ together with a collection $\left\{X_{\alpha}\right\}_{\alpha \in \mathcal{L}(P)}$ of submodules of $X$ such that

$$
X_{\alpha \cap \beta}=X_{\alpha} \cap X_{\beta}, \quad X_{\alpha \cup \beta}=X_{\alpha}+X_{\beta}, \quad X_{\emptyset}=\{0\}, \quad X_{P}=X .
$$

A $P$-graded module is a module $X$ together with a direct sum decomposition

$$
X=\bigoplus_{p \in P} X_{p}
$$

Each subset $\alpha \subset P$ then determines a submodule

$$
X_{\alpha}=\bigoplus_{p \in \alpha} X_{p}
$$

and the collection $\left\{X_{\alpha}\right\}_{\alpha_{\in} \mathcal{L}(P)}$ is a $P$-filtered module. The following lemma enables us to reverse the process and construct a $P$-graded module from a $P$-filtered module.

Proposition 7.1. For $\alpha, \beta \in \mathcal{L}(P)$ with $\alpha \subset \beta$, the quotient

$$
Q_{\beta \backslash \alpha}=X_{\beta} / X_{\alpha}
$$

is a well-defined function of $\beta \backslash \alpha$ up to canonical isomorphism. In particular the quotient

$$
Q_{p}=X_{\beta} / X_{\alpha}, \quad \beta \backslash\{p\}=\alpha
$$


is well defined: the direct sum $Q=\bigoplus_{p \in P} Q_{p}$ thus defined is called the P-graded module determined by the $P$-filtered module $X$.

Proof. Let $\alpha_{j}, \beta_{j} \in \mathcal{L}(P)$ for $j=1,2$ such that $\alpha_{j} \subset \beta_{j}$ and $\beta_{1} \backslash \alpha_{1}=\beta_{2} \backslash \alpha_{2}$. The Lemma asserts that there is a canonical isomorphism

$$
X_{\beta_{1}} / X_{\alpha_{1}}=X_{\beta_{2}} / X_{\alpha_{2}} .
$$

In case $\alpha_{1} \subset \alpha_{2}$ and $\beta_{1} \subset \beta_{2}$ the canonical isomorphism is

$$
\begin{aligned}
X_{\beta_{2}} / X_{\alpha_{2}} & =\left(X_{\beta_{1}}+X_{\alpha_{2}}\right) / X_{\alpha_{2}} \\
& =X_{\beta_{1}} /\left(X_{\beta_{1}} \cap X_{\alpha_{2}}\right) \\
& =X_{\beta_{1}} / X_{\alpha_{1}} . \square
\end{aligned}
$$

Remark. Note that in general the module $Q$ is not isomorphic to $X$ although this is true when the modules $Q_{p}$ are free. In this case there are submodules $X_{p} \subset X$ such that

$$
X_{\alpha}=\bigoplus_{p \in \alpha} X_{p}
$$

for $\alpha \in \mathcal{L}(P)$. We call such a system $\left\{X_{p}\right\}_{p \in P}$ a $P$-splitting for the filtered module $X$. The submodules $X_{p}$ of the $P$-spliting are isomorphic to the quotients $Q_{p}$ but are not unique.

A morphism $F: X \rightarrow X^{\prime}$ between two $P$-filtered modules is called filtration preserving iff

$$
F\left(X_{\alpha}\right) \subset X_{\alpha}^{\prime}
$$

for $\alpha \in \mathcal{L}(P)$. A module homomorphism of $P$-graded modules is determined by its components

$$
F_{p q}: X_{q} \rightarrow X_{p}^{\prime}
$$

with respect to the corresponding direct sum decompositions; $F$ is filtration preserving iff they satisfy

$$
F_{p q} \neq 0 \quad \Longrightarrow \quad p \preccurlyeq q .
$$

A $P$-filtered chain complex consists of a $P$-filtered module $X$ together with a boundary homomorphism $d: X \rightarrow X$ (that is $d^{2}=0$ ) such that $d$ preserves the filtration. A $P$-chain map $F: X \rightarrow X^{\prime}$ of $P$-filtered chain complexes is a module homomorphism which intertwines both structures:

$$
F\left(X_{\alpha}\right) \subset X_{\alpha}^{\prime}, \quad d^{\prime} \circ F=F \circ d .
$$

Two $P$-chain maps $F_{0}, F_{1}: X \rightarrow X^{\prime}$ are called $P$-chain homotopic iff there is a module homomorphism $\Gamma: X \rightarrow X^{\prime}$ which preserves the filtrations and satisfies

$$
F_{1}-F_{0}=d^{\prime} \circ \Gamma+\Gamma \circ d .
$$

Two $P$-filtered chain complexes $X$ and $X^{\prime}$ are called $P$-chain equivalent if there are filtration preserving chain maps $F: X \rightarrow X^{\prime}$ and $G: X^{\prime} \rightarrow X$ such that both $F \circ G: X^{\prime} \rightarrow X^{\prime}$ and $G \circ F: X \rightarrow X$ are chain homotopic to the identity. A $P$-filtered chain complex $X$ determines a collection of homology exact triangles

$$
H\left(X_{\beta} / X_{\alpha}\right) \stackrel{i}{\rightarrow} H\left(X_{\gamma} / X_{\alpha}\right) \stackrel{p}{\rightarrow} H\left(X_{\gamma} / X_{\beta}\right) \stackrel{d}{\rightarrow} H\left(X_{\beta} / X_{\alpha}\right)
$$

for any three lower sets $\alpha \subset \beta \subset \gamma$. These are also called homology braids. 
Proposition 7.2. The homology spaces $H\left(X_{\beta} / X_{\alpha}\right)$ depend only on $\beta \backslash \alpha$ up to canonical isomorphism. The maps $i, p, d$ in the homology braid intertwine these canonical isomorphisms. A P-chain map F induces homology homomorphisms $F_{\beta \backslash \alpha}: H\left(X_{\beta} / X_{\alpha}\right) \rightarrow H\left(X_{\beta}^{\prime} / X_{\alpha}^{\prime}\right)$ which intertwine the maps in the braids. Two $P$-chain maps which are $P$-chain homotopic induce the same homology homomorphisms.

A chain representation for the attractor network $\left(M, f,\left\{A_{\alpha}\right\}_{\alpha \in \mathcal{L}(P)}\right)$ consists of a $P$-filtered chain complex $X$, a $P$-chain automorphism $F: X \rightarrow X$, and a collection of isomorphisms

$$
\Psi_{\beta \backslash \alpha}: H\left(X_{\beta} / X_{\alpha}\right) \rightarrow \mathcal{H}\left(\Lambda_{\beta \backslash \alpha}\right)
$$

which satisfy the following conditions.

(1) The maps $\Psi_{\beta \backslash \alpha}$ form an isomorphism of homology braids. This means that the following diagram commutes for any three lower sets $\alpha \subset \beta \subset \gamma$

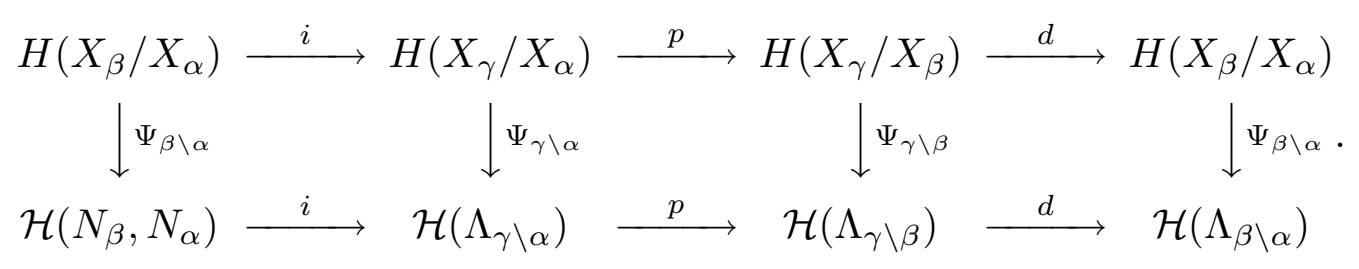

(2) The isomorphism $\Psi_{\beta \backslash \alpha}$ intertwines the induced automorphism for any two lower sets $\alpha \subset \beta$ :

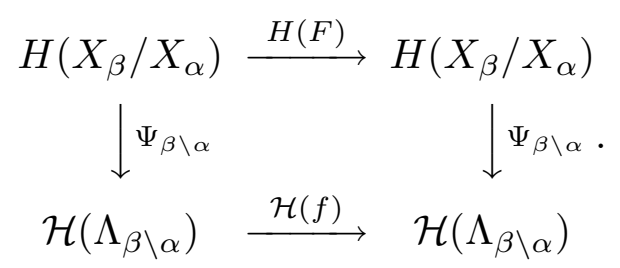

Theorem 7.3. Any attractor network for a smooth dynamical system admits a chain representation. In case the coefficient ring is a field there is a chain repesentation whose underlying vector space is finite dimensional.

Proof. Let $\left\{N_{\alpha}\right\}_{\alpha \in \mathcal{L}(P)}$ a piecewise smooth neighborhood network defining the attractor network as in Theorem 5.1. Using the method of Cairns [1] we can triangulate the manifold $M$ so that the subsets $N_{\alpha}$ are subcomplexes. Denote the resulting network of simplicial subcomplexes by $K_{\alpha}$. As in Spanier [21], chapter 3, choose a subdivision $K^{\prime}$ and a simplicial approximation $\phi: K^{\prime} \rightarrow K$ to $f$. Denote the subdivision map on simplicial chains by $\tau: C(K) \rightarrow C\left(K^{\prime}\right)$. The composition

$$
\Phi=C(\phi) \circ \tau: C(K) \rightarrow C(K)
$$

is a $P$-chain map which induces $H(f)$ on homology.

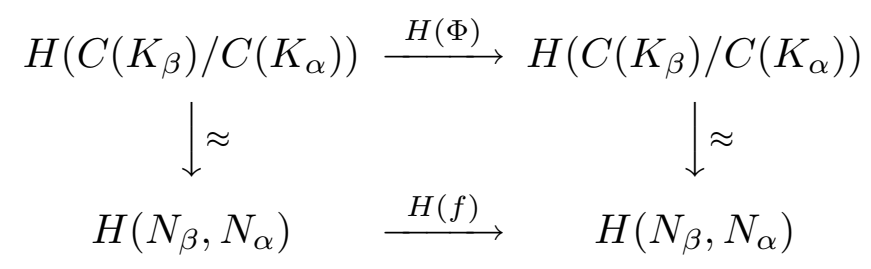


Let

$$
X=\lim (C(K), \Phi)
$$

denote the direct limit of the direct system obtained by iterating $\Phi$ and let $F$ be the shift automorphism on the limit. When the coefficient ring is a field

$$
X=C(K) / \operatorname{ker} \Phi^{n}
$$

for sufficiently large $n$ and $F$ is the automorphism of $X$ induced by $\Phi$. The limit is a chain complex since it is a limit of chain complexes. Since the homology of the limit is naturally isomorphic to the limit of the homology it follows that there is a commuting diagram

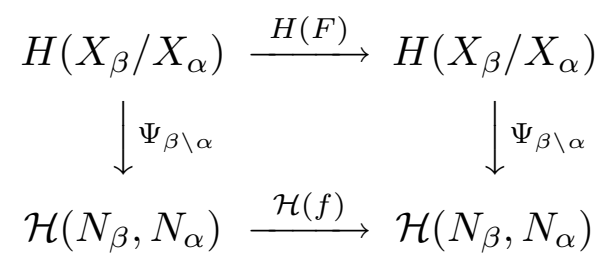

This proves Theorem 7.3.

Conjecture 7.4. Assume the coefficient ring is a field. Then any two chain repesentations of the same attractor network are P-chain homotopy equivalent. The $P$-chain equivalence intertwines the respective automorphisms up to $P$-chain homotopy.

\section{$\S 8$ Connection matrices}

A $P$-connection matrix is a $P$-chain complex $(C, \Delta)$ with the property that

$$
\Delta\left(C_{\beta}\right) \subset C_{\beta \backslash\{p\}}
$$

whenever $p$ is maximal in $\beta$. This means that

$$
H\left(Q_{p}\right)=Q_{p}
$$

where $Q_{p}$ is the quotient $C_{\beta} / C_{\alpha}, \alpha=\beta \backslash\{p\}$, and the boundary map on the quotient is induced by $\Delta$. If

$$
C=\bigoplus_{p \in P} C_{p}
$$

is a $P$-splitting of $C$ then a module homomorphism $\Delta: C \rightarrow C$ is a connection matrix if and only if

$$
\begin{gathered}
\Delta^{2}=0, \\
\Delta_{p q} \neq 0 \Longrightarrow \quad p \preccurlyeq q, \\
\Delta_{p p}=0,
\end{gathered}
$$

for $p, q \in P$ where $\Delta_{p q}: C_{q} \rightarrow C_{p}$ are the components of $\Delta$ in the direct sum decomposition. This is the definition given by Franzosa. Thus our corollary below is closely related to the theorem of Franzosa [10] to the effect that any homology braid is isomorphic to the braid determined by a connection matrix. 
Theorem 8.1. For any P-chain complex $X$ over a field there exist subspaces $\left\{X_{p}\right\}_{p \in P},\left\{V_{p}\right\}_{p \in P},\left\{C_{p}\right\}_{p \in P}$ with the following properties:

(1) The subspaces $\left\{X_{p}\right\}_{p \in P}$ form a P-splitting of $X$.

(2) Each $X_{p}$ splits as

$$
X_{p}=V_{p} \oplus C_{p} \oplus d V_{p}
$$

(3) The boundary map $d$ maps $V_{p}$ isomorphically to $d V_{p}$.

(4) The subspace $C=\oplus_{p \in P} C_{p}$ is a P-filtered subcomplex of $X$ and a connection matrix.

Proof. Suppose by induction that the subspaces $X_{p}, V_{p}$, and $C_{p}$ have been constructed for all $p \in \gamma$ for some lower set $\gamma \in \mathcal{L}(P)$ and that they satisfy conditions (1)-(4). Choose $r \in P \backslash \gamma$ such that $\delta=\gamma \cup\{r\}$ is a lower set. We must construct $X_{r}, V_{r}$, and $C_{r}$ satisfying:

$$
\begin{gathered}
X_{\delta}=X_{\gamma} \oplus X_{r}, \\
X_{r}=V_{r} \oplus C_{r} \oplus d V_{r}, \\
V_{r} \cap d^{-1} 0=0, \\
d C_{r} \subset C_{\rho \backslash\{r\}}
\end{gathered}
$$

where $\rho=\{p \in P: p \preccurlyeq r\}$ is the smallest lower set containing $r$ as a maximal element. We first choose $V_{r}$ to be any complement to $d^{-1} X_{\gamma}$ in $X_{\delta}$ :

$$
X_{\delta}=V_{r} \oplus X_{\delta} \cap d^{-1} X_{\gamma}
$$

Such a complement must satisfy $d V_{r} \cap X_{\gamma}=0$. We will then find a complement $C_{r}$ to $d V_{r} \oplus X_{\gamma}$ in $X_{\delta} \cap d^{-1} X_{\gamma}$ :

$$
X_{\delta} \cap d^{-1} X_{\gamma}=C_{r} \oplus d V_{r} \oplus X_{\gamma}
$$

This complement will also satisfy

$$
C_{r} \subset X_{\rho} \cap d^{-1} C_{\gamma}
$$

Note that (8-5)-(8-7) imply (8-1)-(8-4).

We first prove

$$
V_{\gamma} \cap\left(C_{\gamma}+d X_{\delta}\right)=0
$$

Let $v_{\gamma} \in V_{\gamma}, c_{\gamma} \in C_{\gamma}$, and $x_{\delta} \in X_{\delta}$ such that $v_{\gamma}=d x_{\delta}+c_{\gamma}$. Then $d v_{\gamma}=d c_{\gamma} \in C_{\gamma}$, hence $d v_{\gamma}=0$, and hence $v_{\gamma}=0$.

Now we prove

$$
X_{\delta} \cap d^{-1} X_{\gamma}=X_{\gamma}+X_{\rho} \cap d^{-1} C_{\gamma}
$$


This will justify the choice of $C_{r}$ satisfying (8-6) and (8-7). It is obvious that the right hand side of (8-9) is a subset of the left hand side. For the reverse inclusion choose $x_{\delta} \in X_{\delta}$ with $d x_{\delta} \in X_{\gamma}$. By the lattice property $X_{\delta}=X_{\gamma}+X_{\rho}$ so $x_{\delta}=x_{\gamma}+x_{\rho}$ with $x_{\gamma} \in X_{\gamma}, x_{\rho} \in X_{\rho}$. Then $d x_{\rho} \in X_{\gamma} \cap X_{\rho}=X_{\rho \backslash\{r\}}$. By the induction hypothesis write

$$
d x_{\rho}=v+c+d w
$$

where $v, w \in V_{\rho \backslash\{r\}}$ and $c \in C_{\rho \backslash\{r\}}$. Then $v=d\left(x_{\rho}-w\right)-c=0$, by (8-8). Hence $d\left(x_{\rho}-w\right)=c \in C_{\gamma}$ so

$$
x_{\rho}=w+\left(x_{\rho}-w\right) \in X_{\gamma}+X_{\rho} \cap d^{-1} C_{\gamma} .
$$

Thus we have proved (8-9).

Remark. In case $P$ is a total ordering Theorem 8.1 is an easy consequence of Zeeman's theory of spectral sequences [25].

Corollary 8.2. Every P-chain complex over a field is P-chain equivalent to a $P$-connection matrix.

Proof. Let $\iota: C \rightarrow X$ be the inclusion and $\pi: X \rightarrow C$ be the projection along $V \oplus d V$. Then $\pi \circ \iota=\mathrm{id}_{C}$ and

$$
\iota \circ \pi-\mathrm{id}_{X}=\Gamma d+d \Gamma
$$

where $\left.\Gamma\right|_{d V}=\left(\left.d\right|_{V}\right)^{-1}: d V \rightarrow V$ and $\left.\Gamma\right|_{V \oplus C}=0$.

Corollary 8.3. Assume the coefficient ring is a field. Then any attractor network for a smooth dynamical system admits a chain representation which is a connection matrix.

Proof. Let $(X, d, F, \Psi)$ be the chain representation for the attractor network constructed in the proof of Theorem 7.3. By Theorem 8.1 the $P$-chain complex $(X, d)$ decomposes as

$$
X=V \oplus C \oplus d V
$$

such that $\left.d\right|_{V}: V \rightarrow d V$ is a vector space isomorphism, $d C \subset C$, and $\Delta=\left.d\right|_{C}$ is a connection matrix. By Corollary 8.2 the inclusion $\iota: C \rightarrow X$ is a $P$-chain homotopy equivalence whose $P$-chain homotopy inverse is the projection $\pi: X \rightarrow C$. Let

$$
\Phi_{\beta \backslash \alpha}: H\left(C_{\beta} / C_{\alpha}\right) \rightarrow H\left(X_{\beta} / X_{\alpha}\right)
$$

be the isomorphism on homology induced by $\iota$.

The map

$$
G=\pi \circ F \circ \iota: C \rightarrow C
$$

commutes with $\Delta$. Moreover,

$$
F^{\prime} \circ \iota=\iota \circ G
$$

where $F^{\prime}=\iota \circ \pi \circ F: X \rightarrow X$ is $P$-chain homotopic to $F$. This shows that

$$
H(F) \circ \Phi=\Phi \circ H(G)
$$


It follows that the isomorphism

$$
\Psi_{\beta \backslash \alpha} \circ \Phi_{\beta \backslash \alpha}: H\left(C_{\beta} / C_{\alpha}\right) \rightarrow \mathcal{H}\left(\Lambda_{\beta \backslash \alpha}\right)
$$

intertwines $H(G)$ with the shift automorphism $\mathcal{H}(f)$ on $\mathcal{H}\left(\Lambda_{\beta \backslash \alpha}\right)$.

Let $(C, \Delta, F, \Psi)$ be a chain representation for the attractor network $\left\{A_{\alpha}\right\}_{\alpha \in \mathcal{L}(P)}$. If $\Delta: C \rightarrow C$ is a connection matrix then we have

$$
Q_{p}=C_{\beta} / C_{\beta \backslash\{p\}}=H\left(C_{\beta} / C_{\beta \backslash\{p\}}\right)
$$

where $p$ is amximal in $\beta$. Thus $\Psi$ gives an isomorphism from $Q_{p}$ to $\mathcal{H}\left(N_{\beta}, N_{\beta \backslash\{p\}}\right)$. If the coefficient ring is a field then it follows that the connection matrix can be defined on the $P$-filtered vector space

$$
C=\bigoplus_{p \in P} C_{p}, \quad C_{p}=\mathcal{H}\left(N_{\beta}, N_{\beta \backslash\{p\}}\right)
$$

In the continuous-time case $C_{p}=H\left(N_{\beta}, N_{\beta \backslash\{p\}}\right)$ and $F: C \rightarrow C$ is the identity. Thus Corollary 8.3 extends Franzosa's result [8] to the discrete-time case.

Conjecture 8.4. Assume a general ring as coefficient ring. Any attractor network for a smooth dynamical system admits a chain representation which is a connection matrix. (We do not require that $C$ admits a P-splitting.)

Conjecture 8.5. Assume the coefficient ring is a field. Then any two chain representations of the same attractor network are P-chain isomorphic. The P-chain isomorphism intertwines the respective automorphisms. 


\section{$\S 9$ ExAmples}

Example 1 (For Section 1). An example where the set of attractors is infinite is the gradient flow on the interval whose fixed point set is the Cantor set.

Example 2 (For Proposition 1.1). The stable manifold of an attractor is an open neighborhood of the attractor. The converse is false. As an example consider a rotation on the circle with a stop point. The stop point is not an attractor although its stable manifold is the entire circle.

Example 3 (For Theorem 1.2). Let $M$ be the union of the unit circle in the complex plane with the unit interval on the real axis. Define a flow on $M$ consisting of four orbits. 0,1 , a connecting orbit from 1 to 0 on the real interval, and a connecting orbit from 1 to itself on the unit circle. The only nonempty attractors are $A_{0}=\{0\}$ and $A_{01}=M$. The compact isolated invariant sets $\Lambda_{0}=\{0\}$ and $\Lambda_{1}=\{1\}$ satisfy all the requirements of Theorem 1.2 except that $\Lambda_{1}$ is not the intersection of its stable and unstable manifold.

Example 4 (For Theorem 1.2). An isolated invariant set which is the intersection of its stable and unstable manifold need not be a basic set in any attractor network. As an example consider a rotation on the circle with two stop points.

Example 5 (A Lyapunov map). Let $M=\mathbb{C} P^{n}=S^{2 n+1} / S^{1}$ and $f^{t}$ be the gradient flow of the function

$$
H(z)=\sum_{j=0}^{n} \rho_{j}\left|z_{j}\right|^{2}
$$

where $\rho_{0}>\rho_{1}>\cdots>\rho_{n}$. Note that $H$ is invariant under the torus action $(z, x) \mapsto z^{\prime}$ where $z_{j}^{\prime}=e^{2 \pi i x_{j}} z_{j}$. Here we realize the $n$-torus as the quotient $T^{n}=V / \Gamma$ where $V \subset \mathbb{R}^{n+1}$ is the hyperplane

$$
\sum_{j=0}^{n} x_{j}=0
$$

and $\Gamma=V \cap \mathbb{Z}^{n+1}$ is the integer lattice. In this case $P$ is the totally ordered set $P=\{0,1, \ldots, n\}$ and hence $|K(P)|$ is the standard $n$-simplex. A Lyapunov map for this dynamical system is given by

$$
\psi: \mathbb{C} P^{n} \rightarrow \Delta^{n}, \quad\left[z_{0}: z_{1}: \cdots: z_{n}\right] \mapsto\left(\frac{\left|z_{0}\right|^{2}}{|z|^{2}}, \frac{\left|z_{1}\right|^{2}}{|z|^{2}}, \ldots, \frac{\left|z_{n}\right|^{2}}{|z|^{2}}\right) .
$$

This is the moment map of the torus action on the symplectic manifold $\mathbb{C} P^{n}$.

Example 6 (Connection matrices for Morse-Smale gradient flows). Let $f^{t}: M \rightarrow M$ be a Morse-Smale gradient flow on a compact manifold $M$. Then $P$ is the set of critical points with $p \preccurlyeq q$ iff there is a flow line from $q$ to $p$. The Morse-Smale condition implies that if $p \preccurlyeq q$ and $p \neq q$ then $\mu(p)<\mu(q)$ where the map

$$
\mu: P \rightarrow \mathbb{Z}
$$

assigns to each point $p \in P$ its Morse index. The vector space $C_{p}$ is 1-dimensional for every $p$ and hence $C$ is generated by the elements of $P$. In this case the connection matrix $\Delta$ is uniquely determined provided that we identify $C_{p}$ with $H\left(\Lambda_{p}\right)$ 
and require the isomorphism $\Psi_{p}: C_{p}=H\left(C_{p}\right) \rightarrow H\left(\Lambda_{p}\right)$ to be the identity. With coefficients in $\mathbb{Z}_{2}$ one finds that $\Delta_{p q}$ is the number of flow lines from $q$ to $p$, counted modulo 2, whenever $\mu(q)-\mu(p)=1$ and $\Delta_{p q}=0$ otherwise. The homology of this chain complex $(C, \Delta)$ is isomorphic to the homology of $M$. This is the Morse complex as described by Witten [24] (see also [18]).

Example 7 (Connection matrices for Axiom A No-Cycle systems). Consider a diffeomorphism $f: M \rightarrow M$ of a compact manifold $M$ with a hyperbolic chain recurrent set. Then the chain recurrent set agrees with the nonwandering set $\Omega$ and decomposes into finitely many chain transitive components $\Omega_{p}$ indexed by the poset $P$ with the Smale partial order as described in the introduction. The map

$$
\mu: P \rightarrow \mathbb{Z}
$$

assigns to each $p \in P$ the dimension of the unstable bundle $E^{u}$ of the hyperbolic invariant set $\Omega_{p}$. The neighborhood network $\left\{N_{\alpha}\right\}_{\alpha \in \mathcal{L}(P)}$ can be chosen such that the homology of the pair $\left(N_{\beta}, N_{\beta \backslash\{p\}}\right)$ is nonzero only in dimension $\mu(p)$ whenever $p$ is maximal in $\beta$. Thus the $P$-grading of the module

$$
C=\bigoplus_{p \in P} C_{p}, \quad C_{p}=\mathcal{H}\left(N_{\beta}, N_{\beta \backslash\{p\}}\right)
$$

refines the integer grading by $\mu(p)$.

Now assume that the stable and unstable manifolds $W^{s}\left(\Omega_{p}\right)$ and $W^{u}\left(\Omega_{q}\right)$ intersect transversally for all $p, q \in P$. (This is Smale's Axiom B.) Then there is no connecting orbit from $\Omega_{q}$ to $\Omega_{p}$ unless $\mu(p) \leq \mu(q)$. In contrast to the case of a Morse-Smale gradient flow connecting orbits may exist in the case $\mu(q)=\mu(p)$ with $p \neq q$. The connection matrix $\Delta: C \rightarrow C$ constructed in the proof of Corollary 8.3 is of degree -1 :

$$
\Delta_{p q} \neq 0 \quad \Longrightarrow \quad p \preccurlyeq q, \quad \mu(p)=\mu(q)-1 .
$$

The $P$-chain automorphism $F: C \rightarrow C$ is of degree 0 :

$$
F_{p q} \neq 0 \quad \Longrightarrow \quad p \preccurlyeq q, \quad \mu(p)=\mu(q) .
$$

Thus the automorphism $F$ detects 0 -dimensional heteroclinic orbits between different basic sets of the same index whereas the connection matrix $\Delta$ corresponds to 1-dimensional components of connecting orbits between basic sets of index difference 1. Moreover, it follows from the Lefschetz fixed point theorem that the shift automorphism $F_{p p}: C_{p} \rightarrow C_{p}$ is related to the periodic orbits in $\Omega_{p}$ :

$$
\operatorname{trace}\left(F_{p p}{ }^{k}\right)=\sum_{f^{k}(x)=x \in \Omega_{p}} \nu\left(x ; f^{k}\right)=\nu_{k}\left(\Omega_{p}\right)
$$

where $\nu\left(x ; f^{k}\right)= \pm 1$ according to whether the restriction $\left.d f^{k}(x)\right|_{E_{x}^{u}}$ is orientation preserving or orientation reversing. Note that this implies rationality of the homology zeta function:

$$
\frac{1}{\operatorname{det}\left(\nVdash-t F_{p p}\right)}=\exp \left(\sum_{k=1}^{\infty} \frac{\nu_{k}\left(\Omega_{p}\right) t^{k}}{k}\right)=\zeta\left(t, \Omega_{p}\right) .
$$




\section{REFERENCES}

[[1]] S. Cairns, A simple triangulation method for smooth manifolds, Bull. Amer. Math. Soc. 67 (1951), 389-390.

[[2]] I Cohen, J. Jones, G. Segal, To appear.

[[3]] C. Conley, Isolated Invariant Sets and the Morse Index, CBMS Reg. Conf. Series Math., Vol. 38, AMS, Providence, R.I., 1978.

[[4]] ФC. Conley, The gradient structure of a flow, Ergod. Th. \& Dynam. Sys. 8 (1988), 11-26.

[[5]] C. Conley and E. Zehnder, Morse type index theory for flows and periodic solutions for Hamiltonian equations, Comm. Pure Appl. Math. 37 (1984), 207-253.

[[6]] $\$$ Eilenberg and N. Steenrod, Foundations of Algebraic Topology, Princeton, 1952.

[[7]] Folkman, The homology groups of a lattice, J. Math \& Mech. 15 (1966), 631-636.

[[8]] I Franzosa, Index filtrations and the homology index braid for partially ordered Morse decompositions, Trans. AMS 298 (1986), 193-213.

[[9]] I Franzosa, The continuation theory for Morse decompositions and connection matrices, Trans. AMS 310 (1988), 781-803.

[[10]R Franzosa, The connection matrix theory for Morse decompositions, Trans. AMS 311 (1989), 561-592.

[[11]P. Johnstone, Stone Spaces, vol. 3, Cambridge Studies in Advanced Mathematics, 1982.

[[12]]J. Kelly, General Topology, Van Norstrand, 1955.

[[13]M Mrozek, The Morse equation in Conley's index theory for homeomorphisms, preprint, University of Kraków (1988).

[[14]JJ.Falis and W. de Melo, Geometric Theory of Dynamical Systems, Springer-Verlag, New York, 1982.

[[15]JJ. Robbin and D.A. Salamon, Dynamical Systems, shape theory and the Conley index, Ergod. Th. \& Dynam. Sys. 8 (1988), 375-393.

[[16]G . Rota, On the foundations of combinatorial theory, Zeitschrift für Warscheinlichkeitstheorie und Verwandte Gebiete 2 (1964), 340-368.

[[17]P A. Salamon, Connected simple systems and the Conley index of isolated invariant sets, Trans. A.M.S. 291 (1985), 1-41.

[[18]P】Salamon, Morse theory, the Conley index and Floer homology, Bull. L.M.S. 22 (1990), $113-140$.

[[19]G Segal Classifying spaces and spectral sequences, Pub. IHES 34 (1968), 105-112.

[[20]\$. Smale, Differentiable dynamical systems, Bull. Amer. Math. Soc. 73 (1967), 747-817; Reprinted in "The Mathematics of Time", Springer-Verlag, 1980.

[[22]JJ. Pmoller, Shock Waves and Reaction Diffusion Equations, Springer-Verlag, New York, 1983.

[[21]E. Spanier, Shock Waves and Reaction-Diffusion Equations, Springer-Verlag, New York, 1966.

[[23]M] Stone, Topological representation of distributive lattices and Brouwerian logics, Casopis Pest. Mat. Fys. 67 (1937), 1-27.

[[24]E.Witten, Supersymmetry and Morse theory, J. Diff. Geom. 17 (1982), 661-692.

[[25]E. Zeeman, On the filtered differential group, Annals of Math. 66 (1957), 557-585. 